\title{
Routine Quality Control of Clinical Nuclear Medicine Instrumentation: A Brief Review*
}

\author{
Pat Zanzonico \\ Departments of Medical Physics and Radiology, Memorial Sloan-Kettering Cancer Center, New York, New York
}

This article reviews routine quality-control (QC) procedures for current nuclear medicine instrumentation, including the survey meter, dose calibrator, well counter, intraoperative probe, organ ("thyroid") uptake probe, $\gamma$-camera, SPECT and SPECT/CT scanner, and PET and PET/CT scanner. It should be particularly useful for residents, fellows, and other trainees in nuclear medicine, nuclear cardiology, and radiology. The procedures described and their respective frequencies are presented only as general guidelines.

Key Words: instrumentation; PET/CT; SPECT/CT; quality control (QC); scanner; survey meter

J Nucl Med 2008; 49:1114-1131

DOI: 10.2967/jnumed.107.050203

$\mathbf{N}$ uclear medicine is critically dependent on the accurate, reproducible performance of clinical radionuclide counting and imaging instrumentation. Quality control (QC), which may be defined as an established set of ongoing measurements and analyses designed to ensure that the performance of a procedure or instrument is within a predefined acceptable range, is thus a critical component of routine nuclear medicine practice. An extensive series of parameters has been developed over the years for acceptance testing and performance characterization of $\gamma$-cameras, SPECT and PET scanners, and other nuclear medicine instrumentation. And detailed data acquisition and analysis protocols for this purpose have been promulgated by the National Electrical Manufacturers Association (NEMA), the American Association of Physicists in Medicine (AAPM), and other regulatory, advisory, and professional organizations (1-9). In practice, however, less time-consuming and less rigorous procedures often suffice for day-to-day QC. The current article is a brief overview of such routine QC procedures for current nuclear medicine instrumentation,

Received Feb. 13, 2008; revision accepted Apr. 28, 2008.

For correspondence or reprints contact: Pat Zanzonico, Memorial SloanKettering Cancer Center, 1275 York Ave., New York, NY 10021.

E-mail: Zanzonip@mskcc.org

*NOTE: FOR CE CREDIT, YOU CAN ACCESS THIS ACTIVITY THROUGH THE SNM WEB SITE (http://www.snm.org/ce_online) THROUGH JULY 2009. No potential conflict of interest relevant to this article was reported. COPYRIGHT @ 2008 by the Society of Nuclear Medicine, Inc. including the survey meter, dose calibrator, well counter, intraoperative probe, organ ("thyroid") uptake probe, $\gamma$-camera, SPECT and SPECT/CT scanner, and PET and $\mathrm{PET} / \mathrm{CT}$ scanner. The far more rigorous and more extensive acceptance-testing procedures performed for $\gamma$-cameras, SPECT and SPECT/CT scanners, and PET and PET/CT scanners are beyond the scope of this article, which is not intended to supersede or replace manufacturer-recommended acceptance-testing, QC, and preventive-maintenance procedures.

This article should be particularly useful for residents, fellows, and other trainees in nuclear medicine, nuclear cardiology, and radiology. For technical information on the instrumentation covered in this article, including further details on QC procedures, additional reading is recommended (10-17).

\section{SAFETY AND ELECTROMECHANICAL INSPECTION}

For those nuclear medicine instruments that "interface" directly with patients - the intraoperative probe, organ uptake probe, $\gamma$-camera, SPECT and SPECT/CT scanner, and PET and PET/CT scanner-safety features should be regularly inspected. Such features include manual emergencyoff switches ("panic buttons"), collision-detection switches that immediately stop all motion if a collision occurs (e.g., between the rotating $\gamma$-camera detector and the patient during a SPECT acquisition), and interlocks that immediately turn off the x-ray tube of a SPECT/CT or PET/CT scanner if a primary-barrier door is opened during a CT scan. All position displays on the gantry and computer console and all alignment lasers should likewise be visually inspected. All manual motion-control functions (e.g., gantry rotation, detector radial motion, and table translation) should be checked as well. Finally, as with all electromechanical devices, intraoperative probes, organ uptake probes, $\gamma$-cameras, SPECT and SPECT/CT scanners, and PET and PET/CT scanners should be inspected regularly for frayed wires and broken or otherwise damaged electrical insulation, loose electrical or mechanical connections (including missing or visibly loose screws, nuts, or bolts), and dents, sharp edges, or other physical damage. 


\section{Record Keeping}

Documenting of QC procedures and organized recording of the results of such procedures are requirements of a sound, compliant QC program. A written description of all QC procedures, including the acceptable (or tolerance) range of the results of each such procedure and the corrective action for an out-of-tolerance result, should be included in the procedure manual of the facility; for each procedure, the written description should be signed and dated by the facility director, physicist, or other responsible individual. For each QC test performed, the following data, as well as the initials or signature of the individual performing the test, should be recorded on a structured and suitably annotated form: the test performed; the date and time of the test; the make, model, and serial number of the device tested; the make, model, and serial number of any reference sources used; the results of the test; and a notation indicating if the test result was or was not acceptable (i.e., was or was not within the specified tolerance range). Such records should be archived in chronologic order in a secure but reasonably accessible location. It is generally helpful to track the results of QC tests longitudinally (e.g., in the form of a graph of the numeric result vs. date of the test). In this way, long-term trends in instrument performance, often imperceptible from one day to the next, may become apparent. Increasingly, of course, such records are maintained in electronic form (i.e., are computerized). In many jurisdictions, records must still be maintained in hard-copy form - and it is advisable to do so in any case, both as a backup and for convenient review by regulators and other inspectors.

\section{Reference Sources}

In many instances, QC tests of nuclear medicine instrumentation are performed not with the radionuclides that are used clinically but with longer-lived surrogate radionuclides in the form of so-called reference sources. Such standards are commercially available in various activities and geometries, depending on the application. Importantly, the certified activities of such reference sources must be traceable to the National Institute of Standards and Technology (NIST), formerly the National Bureau of Standards. NIST traceability helps ensure the accuracy of the calibrated activity. Because such reference sources are long-lived, a single standard may be used for months to years, avoiding the need to prepare sources on a daily or weekly basis and thereby avoiding possible inaccuracies in dispensing activity as well as the possibility of radioactive contamination. As with all sealed sources, however, reference sources must be checked for leakage of radioactivity (i.e., wipe-tested) at least annually, and an up-to-date inventory of such standards must be maintained. Reference sources are still radioactive at the end of their useful life span and must therefore be returned to the vendor or an authorized third party or otherwise disposed of as radioactive waste.
A long-lived radionuclide comprising a reference source must match, in the frequency and energy of its $x$ - and $\gamma$-ray emissions, the clinical radionuclide for which it acts as a surrogate to ensure that instrument responses to the clinical radionuclide and to its surrogate are comparable. Surrogate radionuclides commonly used in nuclear medicine and their physical properties and applications are summarized in Table 1.

\section{Survey Meters}

Survey meters, an essential component of any radiation safety program, are portable, battery-operated gas-filled ionization detectors or solid-state scintillation detectors used to monitor ambient radiation levels, that is, exposure rates (e.g., in $\mathrm{mR} / \mathrm{h}$ ) or counting rates (e.g., in counts per minute $[\mathrm{cpm}])$. Among ionization-detector survey meters, so-called cutie-pies are relatively low-sensitivity ionization chambers (i.e., are operated at a relatively low potential difference between the anode and cathode) and are designed for use where high fluxes of $\mathrm{x}$ - and $\gamma$-rays are encountered. The more familiar Geiger counters (or GeigerMueller counters) are operated at a high potential difference, providing a high electron amplification factor and thus high sensitivity. Geiger counters are therefore well suited for low-level surveys, for example, checking for radioactive contamination. Generally, cutie-pies are calibrated in exposure rates $(\mathrm{mR} / \mathrm{h})$ and Geiger counters in counting rates (cpm). As an ionization chamber, the cutiepie has an electron signal that depends on the energy of the detected $\mathrm{x}$ - or $\gamma$-rays and is therefore directly related to the exposure for all radionuclides. For Geiger counters, the amplitude of the signal pulses is independent of the energy of the incoming radiation. Therefore, if calibrated for exposure rates $(\mathrm{mR} / \mathrm{h})$, Geiger-counter calibration results apply only to the particular radionuclides comprising the reference source used in the calibration procedure. Solidstate detectors use a non-air-equivalent crystal as the detection medium and thus cannot measure exposure rates; they can measure only counting rates.

QC tests of survey meters generally include a daily battery check, with a display indicating whether the voltage supplied by the battery is within the acceptable operating range. To confirm that the survey meter has not been contaminated (i.e., yields a reproducibly low exposure or counting rate in the absence of radioactivity), the background exposure or counting rate should be measured daily in an area remote from radioactive sources within the nuclear medicine facility, if such an area is reasonably accessible. In addition, survey meters should be checked daily for constancy of response by measuring the exposure or counting rate of a long-lived reference source in a reproducible measurement geometry. Aside from the short-term decay of the reference source, the measured day-to-day exposure or counting rates should agree within $10 \%$; if not, the meter should be recalibrated.

Survey meters should be calibrated - that is, checked for accuracy-using suitable long-lived reference sources at 
TABLE 1

Long-Lived Radionuclides Comprising Reference Sources for Instrumentation QC

\begin{tabular}{|c|c|c|c|c|c|c|}
\hline Radionuclide & Half-life & $\begin{array}{c}\text { Decay } \\
\text { constant }(\lambda)\end{array}$ & $\begin{array}{c}\text { Energy, } E_{\gamma} \\
\text { (frequency), of } \\
\text { principal } \mathrm{X} \text { - or } \\
\gamma \text {-ray (keV) }\end{array}$ & $\begin{array}{c}\text { Specific } \boldsymbol{\gamma} \text {-ray } \\
\text { constant, } \\
\Gamma\left(\mathrm{mR} / \mathrm{h} / \mathrm{cm}^{2} / \mathrm{MBq}\right)\end{array}$ & Geometry and activity & QC application \\
\hline \multirow[t]{3}{*}{${ }^{57} \mathrm{Co}^{*}$} & $272 d$ & $0.00254 / d$ & $122(86 \%)$ & 25.1 & $\begin{array}{l}\text { Test tube-sized rod, } \\
\sim 37 \mathrm{kBq}\end{array}$ & $\begin{array}{l}\text { Well-counter constancy } \\
\text { and accuracy }\end{array}$ \\
\hline & & & & & $\begin{array}{l}\text { Vial/small bottle, } \\
185-370 \mathrm{MBq}\end{array}$ & $\begin{array}{l}\text { Dose-calibrator accuracy } \\
\text { and constancy }\end{array}$ \\
\hline & & & & & $\begin{array}{l}\text { Sheet (up to } 50 \times 60 \mathrm{~cm} \text { ), } \\
370-740 \mathrm{MBq}\end{array}$ & $\gamma$-camera uniformity \\
\hline \multirow[t]{3}{*}{${ }^{68} \mathrm{Ge}^{\star \dagger}$} & $287 d$ & $0.00241 / d$ & $511(178 \%)$ & 14.5 & $\begin{array}{l}\text { Test tube-sized rod, } \\
\sim 37 \mathrm{kBq}\end{array}$ & $\begin{array}{l}\text { Well-counter constancy } \\
\text { and accuracy }\end{array}$ \\
\hline & & & & & $\begin{array}{l}\text { Vial/small bottle, } \\
185-370 \mathrm{MBq}\end{array}$ & $\begin{array}{l}\text { Dose-calibrator accuracy } \\
\text { and constancy }\end{array}$ \\
\hline & & & & & $\begin{array}{l}\text { Cylinder (up to } 5 \mathrm{~L} \text { ), } \\
\text { up to } 370 \mathrm{MBq}\end{array}$ & PET scanner response \\
\hline \multirow[t]{2}{*}{${ }^{133} \mathrm{Ba}^{*}$} & $10.7 y$ & $0.0648 / y$ & $356(62 \%)$ & 64.9 & $\begin{array}{l}\text { Test tube-sized rod, } \\
\sim 37 \mathrm{kBq}\end{array}$ & $\begin{array}{l}\text { Well-counter constancy } \\
\text { and accuracy }\end{array}$ \\
\hline & & & & & $\begin{array}{r}\text { Vial/small bottle, } \\
185-370 \mathrm{MBq}\end{array}$ & $\begin{array}{l}\text { Dose-calibrator accuracy } \\
\text { and constancy }\end{array}$ \\
\hline \multirow[t]{2}{*}{${ }^{137} \mathrm{Cs}$} & $30 y$ & $0.0231 / y$ & $662(86 \%)$ & 89.7 & $\begin{array}{l}\text { Test tube-sized rod, } \\
\sim 37 \mathrm{kBq}\end{array}$ & $\begin{array}{l}\text { Well-counter constancy } \\
\text { and accuracy }\end{array}$ \\
\hline & & & & & $\begin{array}{l}\text { Vial/small bottle, } \\
185-370 \mathrm{MBq}\end{array}$ & $\begin{array}{l}\text { Dose-calibrator accuracy } \\
\text { and constancy }\end{array}$ \\
\hline \multicolumn{7}{|c|}{$\begin{array}{l}{ }^{* 57} \mathrm{Co},{ }^{68} \mathrm{Ge} \text {, and }{ }^{133} \mathrm{Ba} \text { are sometimes known as "mock" } 99 \mathrm{mTc}\left(\mathrm{E}_{\gamma}=140 \mathrm{keV}\right),{ }^{18} \mathrm{~F}\left(\mathrm{E}_{\gamma}=511 \mathrm{keV}\right) \text {, and }{ }^{131} \mathrm{I}\left(\mathrm{E}_{\gamma}=364 \mathrm{keV}\right) \text {, respectivel) } \\
\left.{ }^{668} \mathrm{Ge} \text { in sealed source is in secular equilibrium with its short-lived, positron-emitting daughter, }{ }^{8} \mathrm{Ga} \text { (half-life, } 68 \mathrm{~min}\right) . \\
\text { Information in Table } 1 \text { is based on data from Johns and Cunningham (30). }\end{array}$} \\
\hline
\end{tabular}

installation, annually, and after any repair. If the source is small (compared with the mean free path of its emitted $\mathrm{x}$ - and $\gamma$-rays within the material comprising the source) and the distance between the source and meter large (compared with the dimensions of the source), then a point-source geometry is approximated and the expected exposure rate (in $\mathrm{mR} / \mathrm{h}$ ), $\dot{\mathrm{X}}$, in air is given by the inversesquare law:

$$
\dot{\mathrm{X}}=\frac{\mathrm{A}_{\mathrm{o}} \mathrm{e}^{-\lambda \Delta \mathrm{t}} \Gamma}{\mathrm{d}^{2}},
$$

Eq. 1

where $A_{o}$ is the activity (in $\mathrm{MBq}$ ) of the reference source at calibration, $\lambda$ is the physical decay constant (in /d) of the radionuclide comprising the reference source, $\Delta \mathrm{t}$ is the time interval (in $\mathrm{d}$ ) between the calibration of the reference source and the current measurement, $\Gamma$ is the specific $\gamma$-ray constant (in $\mathrm{mR} / \mathrm{h} / \mathrm{cm}^{2} / \mathrm{MBq}$ ) of the radionuclide comprising the reference source, and $\mathrm{d}$ is the distance (in $\mathrm{cm}$ ) between the reference source and the meter. The referencesource activity should be sufficient to yield an exposure rate of $\sim 1,000 \mathrm{mR} / \mathrm{h}$ under the foregoing measurement conditions, and the exposure rates should be measured on each scale and, by appropriate adjustment of the source-meter distance, at 2 readings ( $\sim 20 \%$ and $\sim 80 \%$ of the maximum) on each scale. For all readings, the expected and measured exposure rates should agree within $10 \%$.
Many nuclear medicine facilities have their survey meters calibrated by the institutional radiation safety office or by a commercial calibration laboratory. In addition to a calibration report (typically a 1-page document) specifying the reference sources used, the measurement procedure, and the measured and expected exposure rates, a dated sticker summarizing the calibration results should be affixed to the meter itself.

\section{Dose Calibrators}

The dose calibrator is a pressurized gas-filled ionization chamber for assaying activities in radiopharmaceutical vials and syringes and in other small sources. Among routine dosecalibrator QC tests, constancy must be checked daily and accuracy and linearity at least quarterly $(7,18,19)$; daily checks of accuracy are recommended, however. At installation and after service of a dose calibrator, its geometry (position and volume)-dependent response for ${ }^{99 \mathrm{~m}} \mathrm{Tc}$ must be measured and volume (from 2 to $25 \mathrm{~mL}$ )-dependent correction factors relative to the standard volume (e.g., $10 \mathrm{~mL}$ ) derived.

For the constancy test, a reference source, such as ${ }^{57} \mathrm{Co}$, ${ }^{133} \mathrm{Ba},{ }^{68} \mathrm{Ge}$, or ${ }^{137} \mathrm{Cs}$ (Table 1), is placed in the dose calibrator, and the activity reading on each scale is recorded; day-to-day readings should agree within $10 \%$. For the accuracy test (also sometimes known as the energy linearity 
test), NIST-traceable reference sources of at least 2 of the radioisotopes listed in Table 1 are separately placed in the dose calibrator and the activity reading on each scale recorded. For each source, the measured activity on each scale and its current actual activity should agree within $10 \%$.

For the quarterly check of linearity by the so-called decay method, one begins with a high-activity $(\sim 37$ $\mathrm{GBq}$ ), independently calibrated ${ }^{99 \mathrm{~m}} \mathrm{Tc}$ source and assays its activity at 12-h intervals over 3 consecutive days. Over that time, which is equivalent to 12 half-lives of ${ }^{99 \mathrm{~m}} \mathrm{Tc}$, the activity decays to about $11 \mathrm{MBq}$. The measured activities are then plotted versus time on a semilogarithmic graph, and the best-fit straight line drawn through the data points is plotted (either by eye or by using a least-squares curvefitting algorithm). For each data point, the difference between the measured activity and the activity on the best-fit straight line at that point should be less than $10 \%$. An alternative approach to checking linearity is the so-called shield method, in which lead sleeves of increasing thickness are placed in the dose calibrator with a ${ }^{99 \mathrm{~m}}$ Tc source (Fig. 1). By interposing increasing decayequivalent thicknesses (as specified by the manufacturer of the set of lead sleeves) between the source and the sensitive volume of the dose calibrator, a decay-equivalent activity is measured for each sleeve. Although the shield method is much faster than the decay method for checking linearity (taking minutes instead of days), an initial decaybased calibration of the set of sleeves is recommended to accurately determine the actual decay equivalence of each shield.

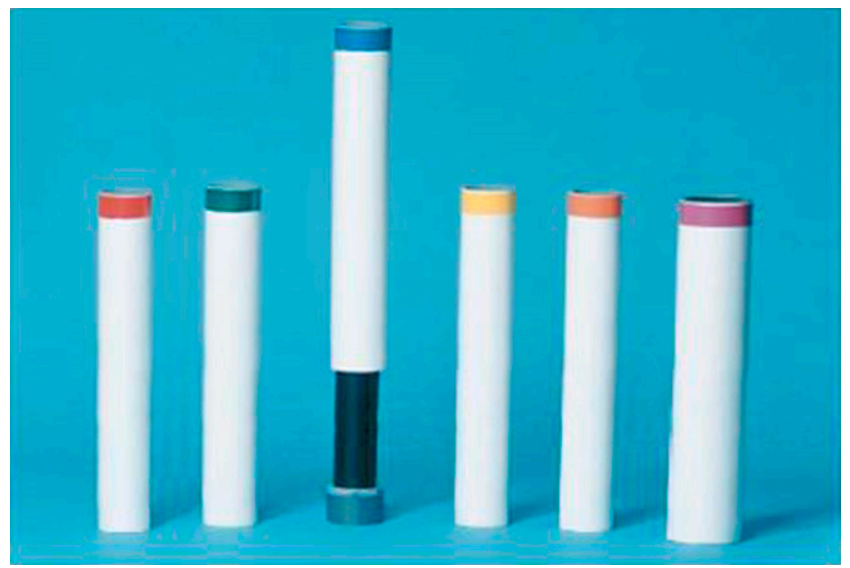

FIGURE 1. Set of lead-lined plastic sleeves (CalicheckDose Calibrator Linearity Test Kit; Fluke Biomedical) for evaluation of dose-calibrator linearity by shield method. Set is supplied with 0.64-cm-thick lead base, color-coded unlined sleeve (to provide activity measurement equivalent to 0 time point measurement of decay method), and 6 color-coded lead-lined sleeves providing attenuation factors nominally equivalent to decay over $6,12,20,30,40$, and $50 \mathrm{~h}$, respectively, for ${ }^{99 \mathrm{~m} T c}$.

\section{Well Counters}

Well counters are used for high-sensitivity counting of radioactive specimens such as blood or urine samples or "wipes" from surveys of removable contamination (i.e., wipe testing). Such counting results should be expressed in activity (e.g., $\mu \mathrm{Ci}$ ), using the appropriate isotope-specific calibration factor $(\mu \mathrm{Ci} / \mathrm{cpm})$. Such devices generally comprise a cylindric scintillation crystal (most commonly thallium-doped sodium iodide) with a circular bore (well) for the sample, backed by a photomultiplier tube (PMT) and its associated electronics. Modern scintillation well counters are often equipped with a multichannel analyzer (MCA) for energy (i.e., isotope)-selective counting and an automatic sample changer for unattended counting of multiple samples. Because of their high intrinsic and geometric efficiencies (resulting from the use of a thick crystal and a well-type counting geometry, respectively), well counters are extremely sensitive and, in fact, can reliably be used only for counting activities up to approximately $37 \mathrm{kBq}$; at higher activities, dead-time counting losses become prohibitive and the measured counts inaccurate.

The routine QC tests for well counters include checks of the photopeak energy window (i.e., energy peaking) if the counter is equipped with an MCA and of background, constancy, and efficiency (or sensitivity). Before counting samples containing a particular radionuclide, one should check the energy spectrum to verify that the counter is properly peaked, that is, that the photopeak of the radionuclide coincides with the preset photopeak energy window. Isotope-specific radionuclide counting or imaging with a scintillation detector commonly is done using a $20 \%$ photopeak energy window, equivalent to an energy range of $\mathrm{E}_{\gamma} \pm 10 \%$ (i.e., $0.9-1.1 \mathrm{E}_{\gamma}$ ), where $\mathrm{E}_{\gamma}$ is the $\mathrm{x}$ - or $\gamma$-ray energy of the radionuclide.

For each photopeak energy window used, the background counting rate should be checked daily. The energy resolution (expressed as the percentage full width at half maximum [FWHM] of the photopeak) should be checked at least quarterly using a reference-source radionuclide such as ${ }^{57} \mathrm{Co}$. Electronic noise and ambient radiation levels, which may be relatively high and variable in a nuclear medicine facility, will produce a nonzero and potentially fluctuating background counting rate. Further, even trace contamination of the counting well will produce inaccurately high counting-rate values. Accordingly, a blank (i.e., an empty counting tube or vial) should always be included to determine the current background count. To check constancy, at least 1 NIST-traceable reference source (Table 1) should likewise be counted each day; day-to-day net (i.e., gross minus background) counting rates should agree within $10 \%$.

The $\chi^{2}$ test is a statistical method for checking the (shortterm) reproducibility of a well counter, that is, for checking whether the random variation in a set of counting-rate measurements is consistent with that expected for a Poisson 
distribution (11). The $\chi^{2}$ statistic for a set of $n$ counting-rate measurements of a long-lived reference source having a mean $\overline{\mathrm{N}}$ and a standard deviation SD is:

$$
\chi^{2}=\frac{(n-1) \mathrm{SD}^{2}}{\overline{\mathrm{N}}}
$$

Thus, if $P$ and $1-P$ represent the probabilities that random variations in a set of measurements from a Poisson distribution are, respectively, greater than or equal to or smaller than the calculated $\chi^{2}$ value, a $P$ value (or $1-P$ value) of 0.5 indicates the calculated $\chi^{2}$ value is in the middle of the range expected for a Poisson distribution. Conversely, a $P$ value of less than 0.01 or greater than 0.99 , respectively, indicates that there is only a $1 \%$ chance that a Poisson distribution would yield an $\chi^{2}$ value as large as or as small as that actually calculated. Such very small or very large $P$ values are problematic, therefore, because they indicate a variation in counting-rate measurements inconsistent with a Poisson distribution and are suggestive of a counter malfunction. $\chi^{2}$ values yielding a $P$ value in the range of $0.05-0.95$ are generally considered acceptable. It is advisable to perform $\chi^{2}$ testing at least quarterly. Compendia of critical values of the $\chi^{2}$ statistic, as a function of the number of measurements $(n)$, are widely available in both graphical and tabular forms (11).

For each radionuclide for which a particular well counter is used, the counter should be calibrated-that is, its efficiency (sensitivity) (in $\mathrm{cpm} / \mathrm{Bq}$ ), $\varepsilon$, should be determined-at installation, annually, and after any repair:

$$
\varepsilon=\frac{\dot{C}_{\mathrm{RS}}-\dot{C}_{\mathrm{BG}}}{\mathrm{A}_{\mathrm{o}} \mathrm{e}^{-\lambda \Delta \mathrm{t}}}
$$

where $\dot{\mathrm{C}}_{\mathrm{RS}}$ is the gross (i.e., total) counting rate of the radionuclide source, $\dot{\mathrm{C}}_{\mathrm{BG}}$ is the background counting rate, $\mathrm{A}_{\mathrm{o}}$ is the activity (in $\mathrm{Bq}$ ) of the radionuclide source at calibration, $\lambda$ is the physical decay constant of the radionuclide comprising the reference source, and $\Delta \mathrm{t}$ is the time interval between the calibration of the radionuclide source and the current measurement. The radionuclide source should be a precisely calibrated sample $(\sim 37 \mathrm{kBq}$ in $\sim 1 \mathrm{~mL}$ ) prepared by appropriate dilution and careful aliquoting of an activity of that radionuclide sufficiently large to be accurately assayed in a dose calibrator. Because the $\mathrm{x}$ - or $\gamma$-ray energy and frequency may differ somewhat between a particular radionuclide and its surrogate (i.e., reference-standard) radionuclide (Table 1), the counter efficiencies for that radionuclide and its surrogate may differ slightly as well. At installation, therefore, the calibrated sample of each radionuclide (e.g., ${ }^{99 \mathrm{~m}} \mathrm{Tc}$ ) and its surrogate (e.g., ${ }^{57} \mathrm{Co}$ ) should be counted using the photopeak energy window (e.g., $126-154 \mathrm{keV}$ for ${ }^{99 \mathrm{~m}} \mathrm{Tc}$ ) and the respective efficiencies determined. These will yield a factor that can subsequently be used to convert the current efficiency of the surrogate to the current efficiency of the radionuclide. In this way, annual and other checks of efficiency can be performed using only the surrogate reference sources, without the need to dispense, assay, dilute, and aliquot each radionuclide in clinical use.

The minimum detectable activity (MDA) of each radionuclide for which a particular well counter is used is the activity that increases the gross counts over a counting time $t$ by a value that is significantly greater than that due to random variations in the background counts over $t$. In this context, the conventionally applied criterion for statistical significance is a gross counting rate that exceeds the background counting rate $\left(\dot{\mathrm{C}}_{\mathrm{BG}}\right)$ by at least $3 \mathrm{SDs}(11)$ :

$$
\operatorname{MDA}=\frac{3 \sqrt{\frac{\dot{\mathrm{C}}_{\mathrm{BG}}}{\mathrm{t}}}}{\varepsilon} .
$$

The MDA should be evaluated at least annually.

\section{Intraoperative Probes}

Intraoperative probes (17)—-handheld, highly collimated counting devices (i.e., solid-state scintillation or ionization [i.e., semiconductor] detectors) - are now widely used in the surgical management of cancer, most commonly to more expeditiously identify and localize sentinel lymph nodes and thereby reduce the need for more extensive surgery. In addition, such probes have been used to identify and localize visually occult disease at surgery after systemic administration of a radiolabeled antibody or other tumor-avid radiotracer. In addition to daily battery and background checks (as performed for survey meters), QC tests of intraoperative probes should include a daily bias check for both the primary and any back-up battery to verify that bias voltage (or high voltage) is within the acceptable range. Because intraoperative probes may not provide a display of the energy spectrum, one may not be able to visually check that the probe is properly peaked, that is, that the photopeak coincides with the preset photopeak energy window. The lower counts or counting rates resulting from an inappropriate energy window may therefore go unnoticed. Thus, a long-lived reference source or set of reference sources (such as ${ }^{57} \mathrm{Co},{ }^{133} \mathrm{Ba},{ }^{68} \mathrm{Ge}$, or ${ }^{127} \mathrm{Cs}$ [Table 1]) should be available for daily checks of countingrate constancy; a marked change (e.g., $\pm 10 \%$ ) in the net counting rate from one day to the next may indicate an inappropriate energy window setting or some other technical problem. Ideally, each reference source should be incorporated into some sort of cap that fits reproducibly over the probe so that spurious differences in counting rates due to variations in source-detector geometry are avoided.

\section{Organ Uptake Probes}

Historically, organ uptake probes have been used almost exclusively for measuring thyroid uptake and are thus generally known as thyroid uptake probes. The uptake 
probe is a radionuclide counting system comprising a wideaperture, diverging collimator; a thallium-doped sodium iodide crystal (typically $\sim 5 \mathrm{~cm}$ thick by $\sim 5 \mathrm{~cm}$ in diameter); a PMT and associated electronics; an MCA; and a gantry (stand). Aside from the counting geometry and sensitivity, uptake probes and well counters actually have much in common, and the QC procedures-checks of the photopeak energy window, background, constancy, and efficiency-are therefore analogous. (Please refer to the section "Well Counters" for further detail.) However, efficiency of organ uptake probes should be measured more frequently-for each patient-than for a well counter, so that the probe net counting rates can be reliably converted to thyroid uptake for individual patients.

\section{$\gamma$-Cameras}

The $\gamma$-camera has long been, and remains, the most widely used imaging device in nuclear medicine. The performance parameters most commonly evaluated as part of a routine $\gamma$-camera QC program include uniformity, spatial resolution, spatial linearity, and energy resolution and peaking.

$\gamma$-Camera uniformity may be evaluated either intrinsically (i.e., without collimation) or extrinsically (i.e., with collimation) (Fig. 2). Intrinsically, a point source $(<1 \mathrm{~mL}$ in volume and containing $\sim 18.5 \mathrm{MBq}$ ) of ${ }^{99 \mathrm{~m}} \mathrm{Tc}$ is placed 5 crystal dimensions from and centered over the uncollimated detector to provide a near-uniform photon flux impinging on the detector. If necessary, the activity should be adjusted to yield a measured counting rate of no greater than 25,000 cycles per second (cps), to avoid dead-time counting losses and counting-rate-related image degradation. Extrinsically, a uniform flood, or sheet source (typically $185-555 \mathrm{MBq}$ ) of ${ }^{57} \mathrm{Co}$ is placed directly on the collimated detector. A total of 10-15 million counts is acquired and uniformity quantitated for the integral and differential uniformities, which actually express the deviation from uniformity of the flood image. The integral uniformity (IU) is defined by the following equation:

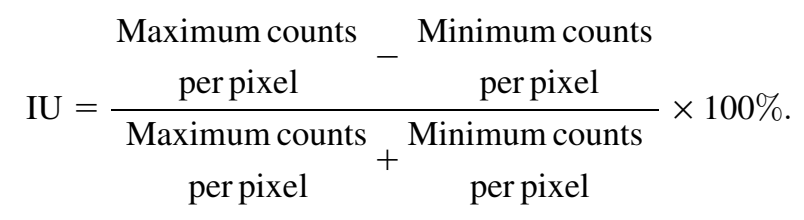

Eq. 5

Differential uniformity (DU) is the maximum value of the expression on the right side of Equation 5 determined for every 5-pixel segment in every row and column of the flood image. IUs of $3 \%$ or better are routinely obtained for modern $\gamma$-cameras. An alternative, and perhaps even more robust, measure of uniformity is the SD of the counts per pixel in the flood image.

If $\gamma$-camera uniformity for any radionuclide is out of tolerance (i.e., IU or DU > 5\%), the uniformity (or sensitivity) correction table of that radionuclide should be updated. As derived from a high-count flood image of a particular radionuclide, the uniformity (or sensitivity) correction table for that radionuclide is essentially the pixel-bypixel ratio of the calculated mean count per pixel to the actual count per pixel in the flood image. By pixel-by-pixel multiplication of a raw (i.e., uncorrected) image by the ratio image thus calculated, an image corrected for the nonuniformity of the $\gamma$-camera is obtained. The necessary data may be acquired using the same setup as used for the daily uniformity test, except that a much larger number of counts $(60-100$ million) must be acquired. In the $\gamma$-cameras of today, uniformity correction tables can be easily updated and the corrections created, processed, stored, and automatically applied using the integrated software of the system. In addition to an outdated uniformity correction table, the following are other causes of $\gamma$-camera nonuniformity (Fig.

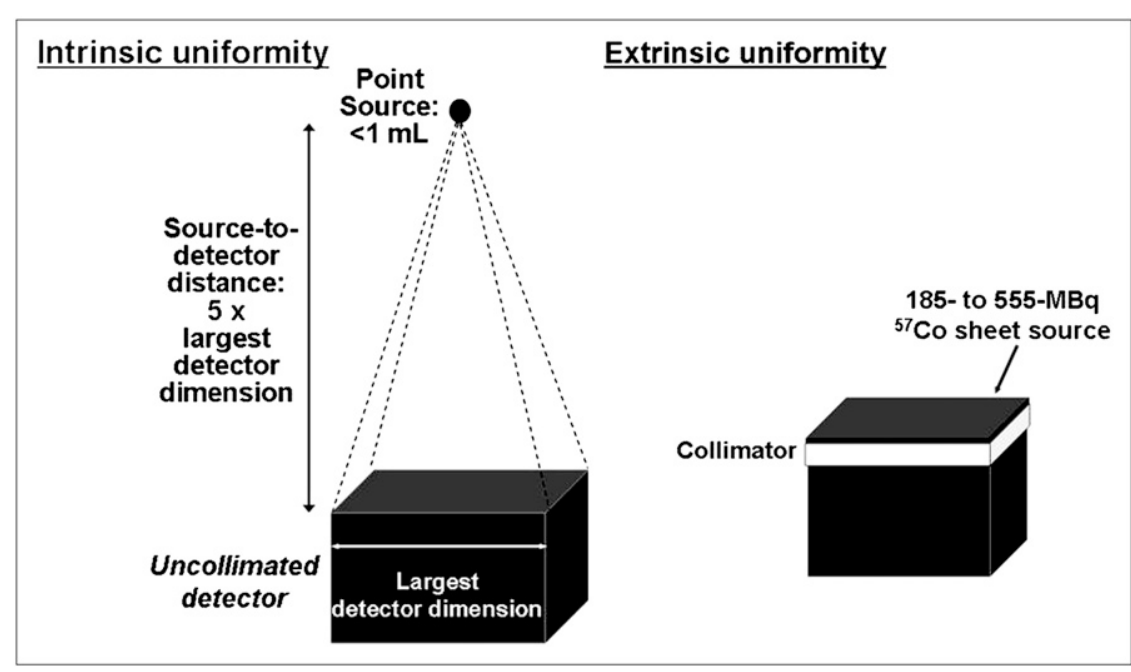

FIGURE 2. $\quad \gamma$-Camera uniformity may be evaluated either intrinsically or extrinsically. Intrinsically, point source is placed at least 3 , and preferably 5, crystal dimensions from and centered over uncollimated detector to provide uniform photon flux (left). Extrinsically, uniform flood, or sheet, source of ${ }^{57} \mathrm{Co}$ is placed directly on collimated detector (right). 


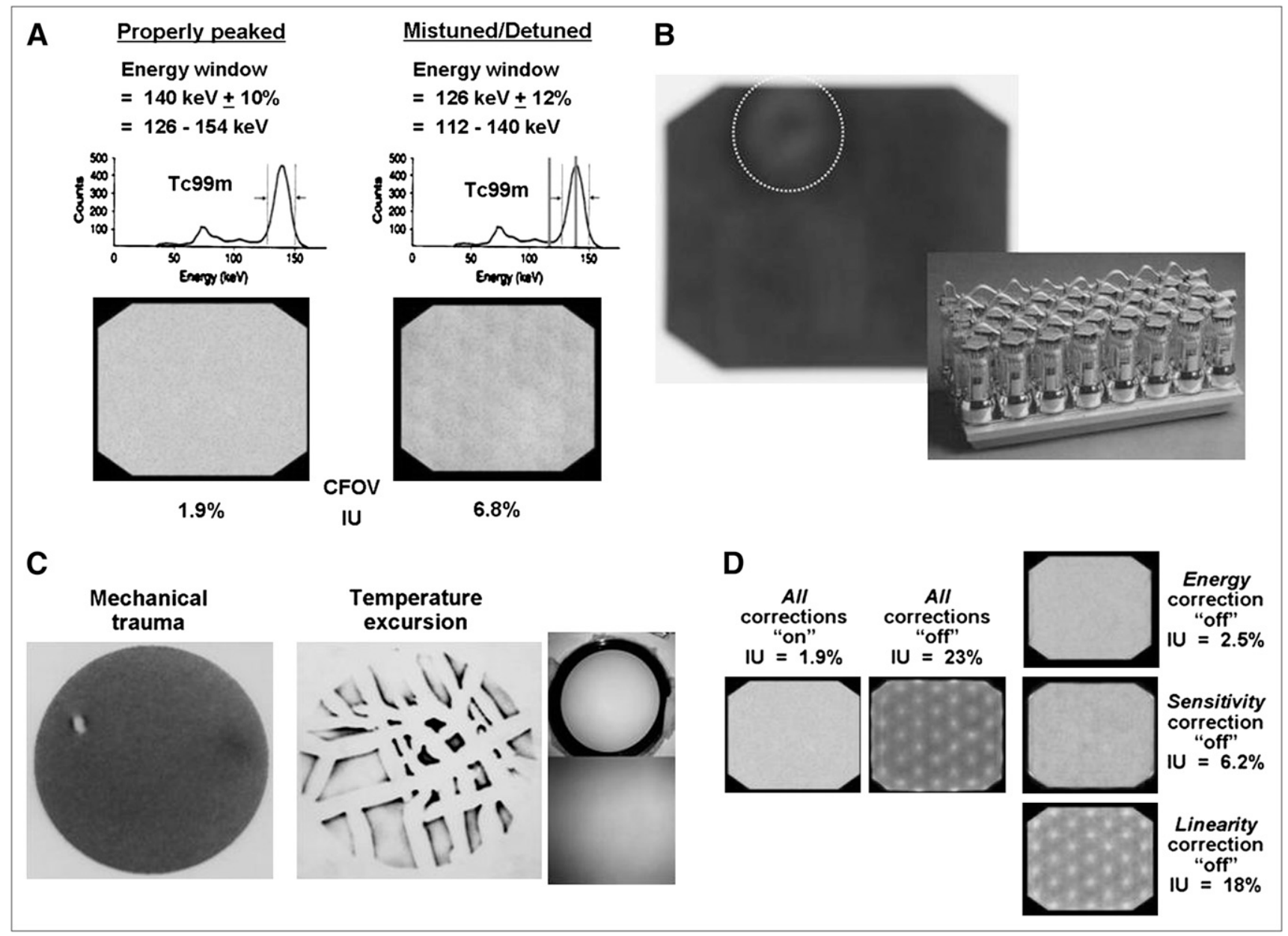

FIGURE 3. Sources of $\gamma$-camera nonuniformity. (A) Mistuning (or detuning), meaning that photopeak of radionuclide does not coincide with photopeak energy window of camera, perhaps because energy window (as shown) or high voltages of PMTs are not set correctly. (B) Uncoupling of PMT from crystal, resulting in loss of all or part of light signal in resulting air gap between PMT entrance window and crystal. (Courtesy of Dr. Barbara Binkert, New York Presbyterian Hospital, New York, NY.) (C) Cracked crystal, either because of mechanical trauma (impact) or temperature excursion (i.e., temperature increase or decrease at rate faster than $\sim 5^{\circ} \mathrm{C}$ per hour, causing crystal to expand or contract, respectively, to point of cracking). Note that it is rate of temperature change that is critical. Photographs on right show cracked crystal that produced corresponding image. Even though cracks are grossly imperceptible, artifacts produced are dramatic. (Courtesy of Dr. Barbara Binkert, New York Presbyterian Hospital, New York, NY.) (D) Corrupted, deleted, or switched-off software correction tables. Even perfectly functioning $\gamma$-cameras have some nonuniformity due to point-to-point variations in energy spectra, greater sensitivity at and lower sensitivity between PMTs, and residual nonuniformity due to ill-defined factors such as variations in crystal thickness. Associated nonuniformities are measured and used to create energy, linearity, and uniformity (or sensitivity) correction tables. Note that linearity correction table has biggest effect on uniformity: if corrupted, deleted, or switched off, PMT pattern becomes grossly apparent, and IU approaches $20 \%$. Fortunately, in contrast to uniformity correction table and, to lesser extent, energy correction table, linearity correction table rarely needs to be updated once $\gamma$-camera is installed; if updating becomes necessary, it is almost always done by field-service personnel of manufacturer, not by end-user.

3): mistuning (detuning), uncoupling of a PMT, a cracked crystal, or corruption or switching off of 1 or more of the correction tables of the camera. The correction tables include uniformity; energy, which essentially corrects the $\gamma$-camera image for nonuniformities related to differences among the local-energy spectra; or linearity, which is largely a geometric correction, correcting the image for nonuniformities related to the position-dependent differences in the efficiency of light collection from the scintillation crystal by the PMTs (i.e., higher efficiency directly beneath the PMTs vs. lower efficiency between the PMTs).
For radionuclides other than ${ }^{99 \mathrm{~m}} \mathrm{Tc}$ used clinically on a particular $\gamma$-camera (i.e., ${ }^{201} \mathrm{Tl},{ }^{123} \mathrm{I},{ }^{111} \mathrm{In},{ }^{67} \mathrm{Ga}$, or ${ }^{131} \mathrm{I}$ ), intrinsic uniformity should be checked at least quarterly.

Spatial resolution and spatial linearity, in practice, are generally assessed semiquantitatively using some sort of resolution phantom (or mask) such as the 4-quadrant bar phantom (Fig. 4A). Such a semiquantitative (i.e., visual) assessment is faster and more convenient than actual measurement of spatial resolution of the FWHM of the line-spread function. A 4-quadrant bar phantom consists of 4 sectors of radioopaque lead bars and intervening radio- 


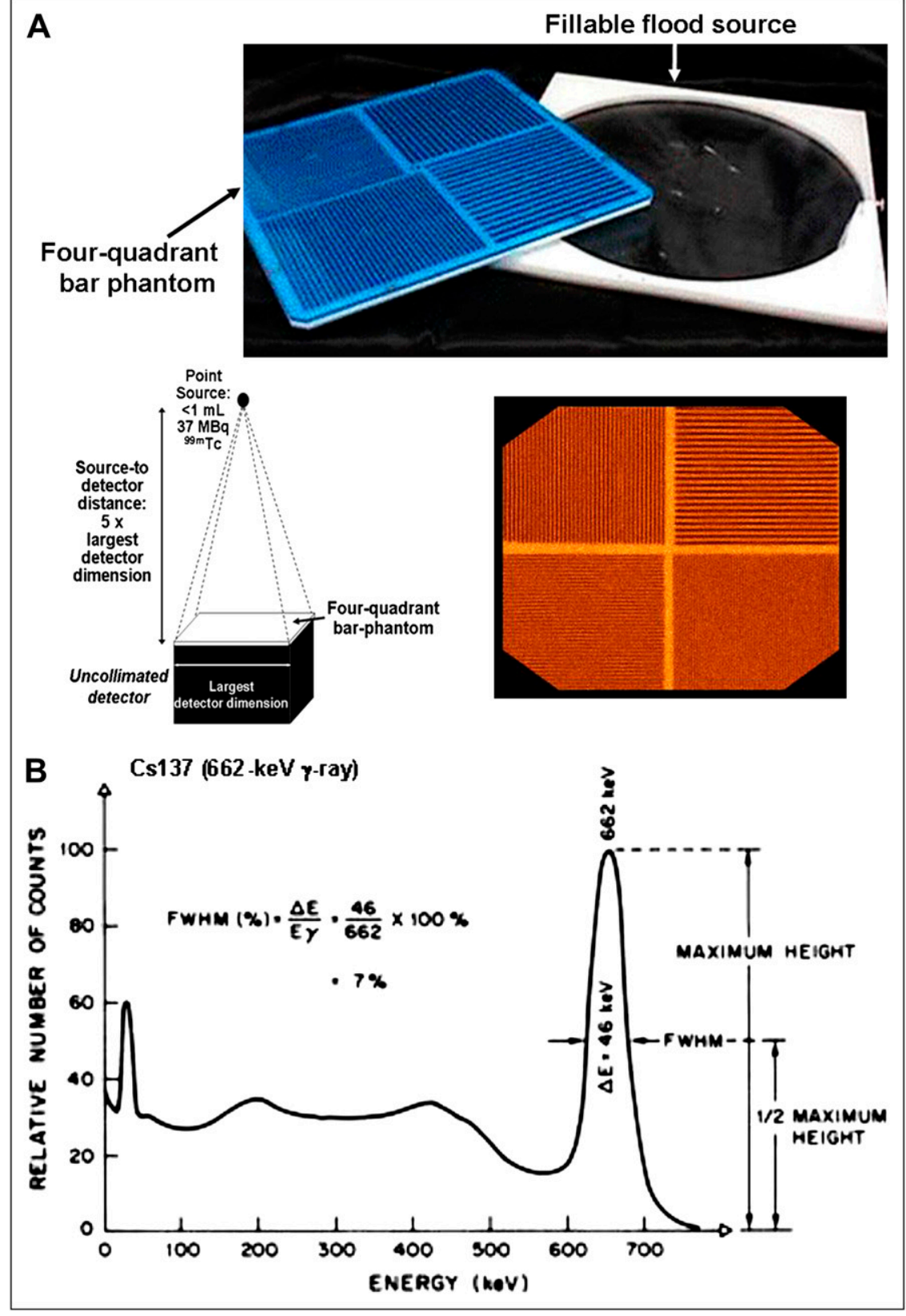

FIGURE 4. (A) Photograph and $\gamma$-camera image of 4-quadrant bar phantom, with schematic diagram illustrating setup for assessment of spatial resolution using such phantom. (Though seldom used in practice, sheet source that may be filled with different radionuclides and used to evaluate extrinsic uniformity is also shown in photograph.) (B) Energy spectrum for $662-\mathrm{keV} \gamma$-ray emitted by ${ }^{137} \mathrm{Cs}$, illustrating definition of energy resolution as percentage FWHM of photopeak en-

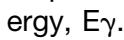

lucent plastic strips 2, 2.5, 3, and $4 \mathrm{~mm}$ in width. A point source of ${ }^{99 \mathrm{~m}} \mathrm{Tc}$ is placed 5 crystal dimensions from and centered over the uncollimated detector, with the phantom placed directly over the detector. A 5- to 10-million-count transmission image is then acquired and visually inspected. The lead bars in at least the 2 coarsest quadrants (i.e., with the 3- and 4-mm-wide bars) should be visually resolvable. Nowadays, at least a portion of the lead bars in the third coarsest quadrant (i.e., with the 2.5-mm-wide bars) should be visible as well. All bars should appear straight. Spatial resolution and spatial linearity should be checked with such a phantom at least weekly.

$\gamma$-Camera energy resolution may be evaluated by the percentage FWHM of the photopeak energy (Fig. 4B). Although energy resolution per se is often not routinely evaluated, the energy spectrum for each radionuclide used clinically should be checked at least once a day and ideally for each patient to verify that the photopeak is centered in the photopeak energy windows currently set; Figure 4A illustrates the type of suboptimal image that may result with a mistuned (detuned) $\gamma$-camera.

\section{Tomographic Image Reconstruction}

Algorithms for reconstruction of tomographic images from projection data-in SPECT as well as in PET_-include analytic techniques such as filtered backprojection and iterative techniques $(11,15,16,20)$. The basic procedure in filtered backprojection is as follows: each projection is Fourier transformed from real to frequency space; the projection is filtered in frequency space using a low-pass 
(e.g., Hanning or Butterworth) filter to eliminate those spatial frequencies above a cutoff frequency and thus reduce statistical uncertainty (or noise); the filtered projection data are inverse Fourier transformed from frequency back to real space; and the filtered projections in real space are uniformly distributed, or backprojected, over the reconstructed image matrix. Iterative algorithms yield progressively refined estimates of the activity distribution, rather than directly calculating the distribution, by maximizing or minimizing some target function. The solution is said to converge when the difference in the target function between successive estimates (iterations) of the activity distribution is less than some prespecified value. Iterative reconstruction algorithms allow incorporation of realistic modeling of the data-acquisition process (including effects of attenuation and of scatter), modeling of statistical noise, and inclusion of pertinent a priori information (e.g., only nonnegative count values). The maximum likelihood expectation maximization (MLEM) algorithm is based on maximizing the logarithm of a Poisson likelihood target function. The MLEM algorithm suppresses statistical noise, but large numbers of iterations typically are required for convergence and, therefore, processing times are long. To accelerate this slow convergence, the ordered-subset expectation maximization (OSEM) algorithm groups the projection data into subsets comprising projections uniformly distributed around the source volume. The OSEM algorithm, which is a modified version of the MLEM algorithm because the target is still maximization of the log likelihood function, converges more rapidly than MLEM and is now the most widely used iterative reconstruction method in PET and SPECT.

\section{SPECT Scanners}

In addition to the QC procedures for $\gamma$-camera imaging listed earlier, rotating- $\gamma$-camera SPECT requires specific QC tests. Among the components of a routine SPECT QC program are periodic assessment of center-of-rotation (COR) alignment, tomographic uniformity, and, for lack of a better term, overall system performance (15). These QC tests apply to both SPECT-only scanners and the SPECT subsystem of SPECT/CT scanners.

In rotating- $\gamma$-camera SPECT, the location of the projection of the COR on the projection image matrix must be independent of the projection-image angle (Fig. 5A). If the mechanical and electronic CORs are aligned, the pixel location of the projection of the COR onto the projection image matrix will be the same for all projection images, and for all such images the counts in each pixel will then be projected across the appropriate row of pixels in the tomographic image matrix. If, however, the mechanical and electronic CORs are not aligned, the pixel location of the COR will vary among the projection images, and the counts in each projection-image pixel will be projected across different locations in the tomographic image matrix and blurred images will result (Fig. 5A). Proper alignment of the mechanical and electronic CORs is therefore critical in rotating- $\gamma$-camera SPECT and should be checked, and if necessary, the COR misalignment correction updated, at least weekly. In current SPECT systems, COR misalignment may be easily measured and corrections created and automatically applied using the integrated software of the system (Fig. 5B).

Because tomographic image reconstruction propagates and, in fact, may amplify the effects of nonuniformities, uniformity of $\gamma$-camera response is another consideration in obtaining high-quality SPECT images. Specifically, the residual, otherwise imperceptible nonuniformity of a $\gamma$-camera with an IU and a DU within tolerance (i.e., <5\%) on the basis of the daily flood image may produce significant ring, or bull's-eye, artifacts in tomographic images (Fig. 6). Tomographic uniformity should therefore be evaluated by high-count imaging of a ${ }^{99 \mathrm{~m}} \mathrm{Tc}$-filled cylinder source (at least $20 \mathrm{~cm}$ in diameter by $20 \mathrm{~cm}$ in length) and visually inspecting the resulting reconstructed images for the absence of perceptible nonuniformity artifacts; this should be done monthly.

Overall system performance may be evaluated using any number of commercially available fillable phantoms containing nonradioactive (cold) inserts of different sizes and visually inspecting the resulting images (Fig. 7). Using such a phantom, so-called cold-sphere contrast may be evaluated on the basis of the minimum counts in sphere $i$ and the mean counts in the plain transverse section closest to the sections through the spheres (Figs. 7D and 7C, respectively (9)):

$\begin{aligned} & \text { Mean counts per pixel } \\ & \text { in the closest uniform }\end{aligned}$
$\begin{gathered}\text { Minimum counts per pixel } \\ \text { transverse section }\end{gathered}$
Mean counts per pixel
in the closest uniform
transverse section

Eq. 6

Graham et al. (9) have provided recommendations on acceptable values of this parameter, ranging from 0.11 to 0.27 for the smallest (i.e., the $15.4-\mathrm{mm}$-diameter) to 0.53 to 0.73 for the largest (i.e., the 31.8-mm-diameter) sphere. Such a phantom also includes a plain section that can be used to simultaneously evaluate tomographic uniformity (Fig. 7C). For such cylinder phantom studies, a ${ }^{99 \mathrm{~m} T c}$ activity of about $10 \mathrm{mCi}$ and at least 64 projections at $40 \mathrm{~s}$ per projection or longer are recommended. Overall SPECT system performance should be checked at least quarterly.

\section{PET Scanners}

As is the case for $\gamma$-cameras and SPECT scanners, detailed protocols for acceptance testing and rigorous evaluation of numerous performance parameters of PET scanners have been developed (4). Additional protocols for acceptance testing of PET and PET/CT scanners are currently being 


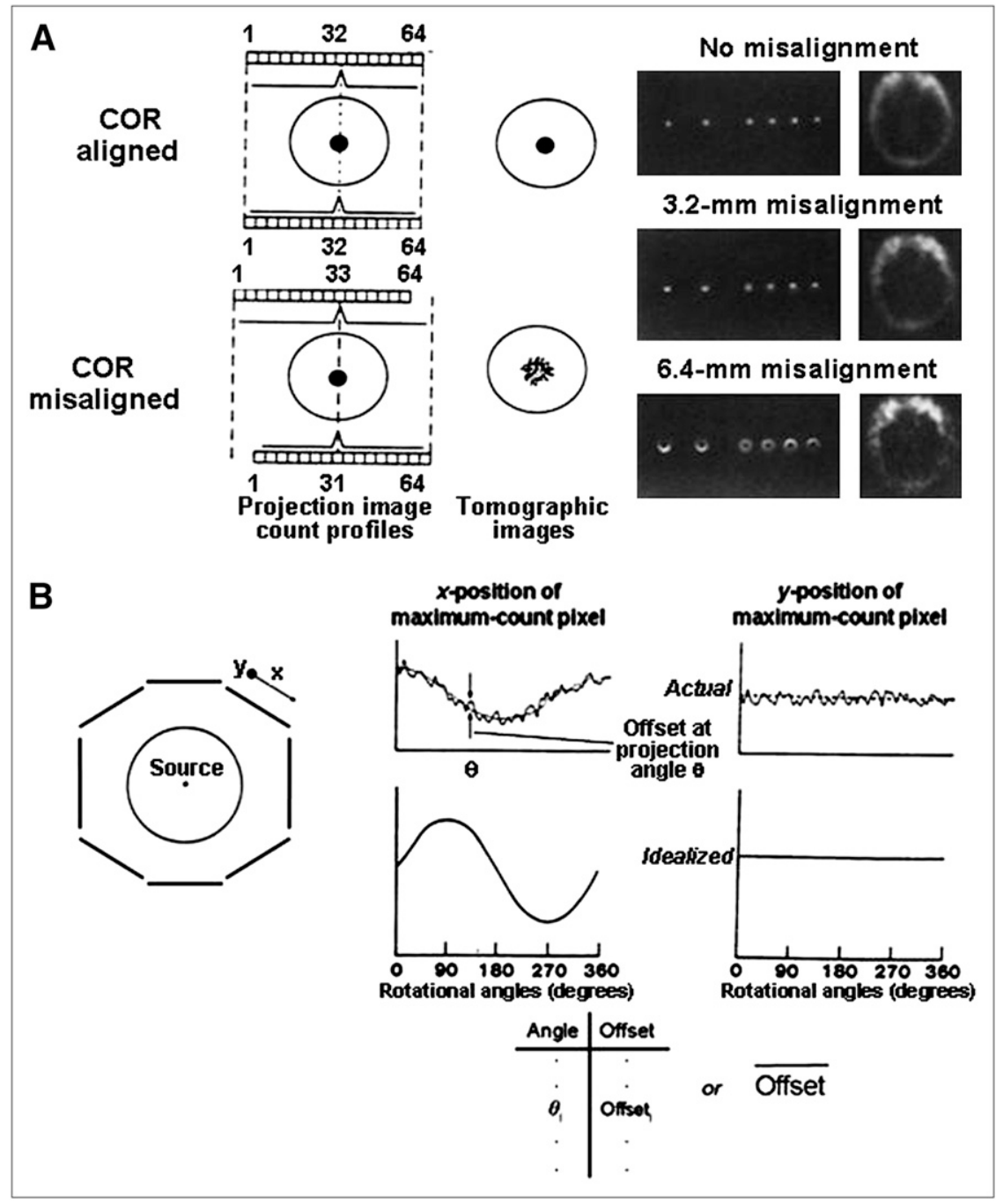

FIGURE 5. (A) COR misalignment and resulting image-blurring artifacts in rotating$\gamma$-camera SPECT. Degree of blurring is related to magnitude of spatial misalignment of mechanical and electronic CORs. Misalignment as small as $3.2 \mathrm{~mm}$ (or 0.5 pixel for $64 \times 64$ image matrix) can produce perceptible blurring in SPECT images, with blurring substantially worse for misalignment of $6.4 \mathrm{~mm}$ (1 pixel). (Adapted from reference (29) with permission.) Note that for cross-sectional image of line source, COR misalignment blurs expected point into full or partial circle depending on position of source in FOV: if it is at or near center of FOV, line source appears as full circle in crosssection; if it is near periphery of FOV, it appears as partial circle. (B) COR misalignment can be measured and corrected on basis of acquiring $360^{\circ}$ circular SPECT study of $99 \mathrm{mTC}$ point source and constructing graphs of $x$ - and $y$-positions (perpendicular and parallel to axis of rotation, respectively) of position of maximum-count pixel in each projection image vs. angular position. $x$ - and $y$-position vs. angle graphs should be sinusoidal curve and straight line, respectively. Angle-by-angle deviation between $x$-position on best-fit sine curve and $x$-position of actual maximum-count pixel thus yields correction table, indicating offset by which each projection image must be shifted at each angular position to align CORs. Alternatively, average of offsets may used at each angular position. (Adapted from reference (15) with permission.)

developed by Task Group 126 of the AAPM and by the International Atomic Energy Agency, respectively.

Less rigorous and less extensive procedures are generally performed for routine QC of PET scanners, however. The most widely used of these are the blank scan and tomographic uniformity. Periodic evaluation and, if necessary, updating of certain calibrations, most notably the normalization and the well-counter calibration, are additional components of routine PET QC. These QC tests and calibrations apply to both PET-only scanners and the PET subsystem of PET/CT scanners.

Blank scans are performed daily by uniformly irradiating the detector elements using either the ${ }^{68} \mathrm{Ge}$ or ${ }^{137} \mathrm{Cs}$ transmission source (if applicable) of the scanner without any source or other attenuating object in the field of view (FOV) or with a uniform source of 511-keV annihilation photons (most commonly a 20 -cm-diameter ${ }^{68} \mathrm{Ge}$ cylinder) centered in the FOV. The term blank scan, derived from the first of these 2 approaches, reflects the absence of any material in the FOV; the term is still often applied to the latter approach, even though it involves placing an object (i.e., the ${ }^{68} \mathrm{Ge}$ source) in the FOV. For PET systems that use ${ }^{68} \mathrm{Ge}$ or ${ }^{137} \mathrm{Cs}$ transmission data to derive attenuation corrections, the blank scan also provides the reference (i.e., unattenuated) transmission data for calculating such corrections. In some respects, the blank scanner is analogous to the daily uniformity flood image acquired for $\gamma$-cameras, providing an overall assessment of detector response.

One approach to evaluating the daily blank scan is simple visual inspection of its (2-dimensional) sinograms (Fig. 8A). In emission tomography (SPECT as well as PET), the emission data are often presented as the 1-dimensional projections (sets of parallel line-integrals) of the direct planes at the azimuthal, or projection, angle $\phi$ relative to the axis of the scanner. The sinogram (or histogram) $(11,16)$ is the full set of 2-dimensional projection data represented as a 2-dimensional matrix in polar coordinates (distance $r$, angle $\phi$ ) in which each row represents the projected intensity across a single direct plane and each column the projected intensity at the same distance $r$ across the projection at successive azimuthal angles $\phi$. The appearance of a blank, or hypointense, diagonal line (Fig. 


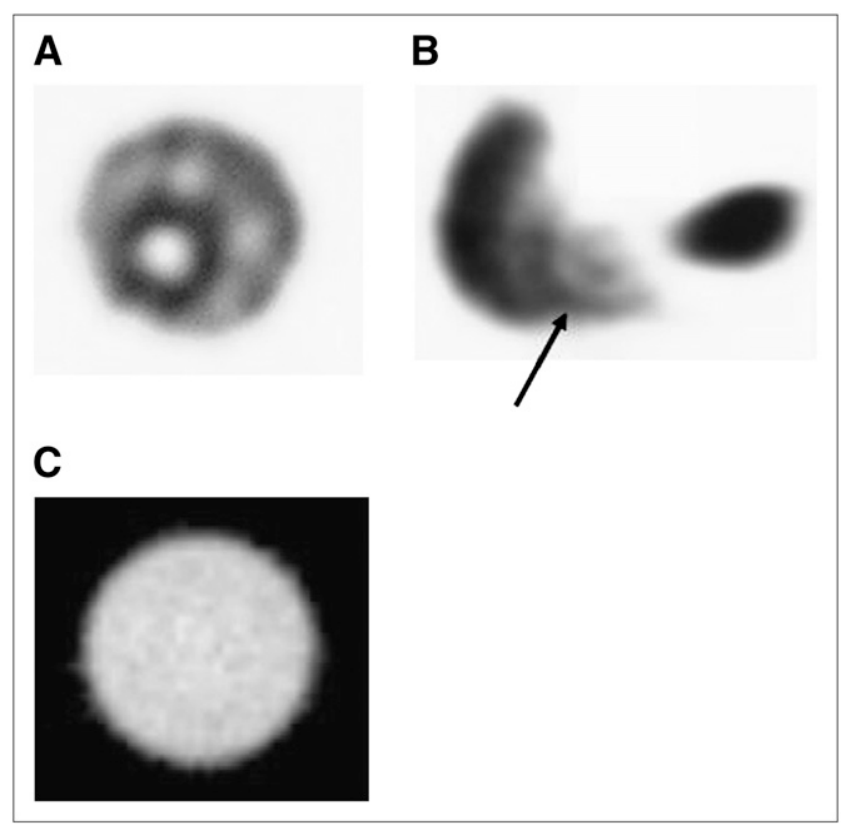

FIGURE 6. (A) Severe ring, or bull's-eye, artifact in transverse SPECT image through $20-\mathrm{cm}$ diameter $99 \mathrm{~m}$ Tc-filled cylinder phantom resulting from gross $\gamma$-camera nonuniformity (i.e., IU $\approx$ $10 \%$ ). (B) Appearance of such artifact (arrow) in clinical SPECT

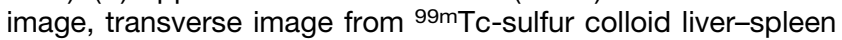
study. (C) In contrast to A, this is acceptable transverse SPECT image through ${ }^{99 m}$ Tc-filled cylinder phantom. No perceptible ring, or bull's-eye, artifact is demonstrated; IU at time of this acquisition, $\sim 2 \%$, was well within tolerance. In cylinder phantom images, $\mathrm{A}$ and $\mathrm{B}$, analytic postprocessing attenuation correction was applied.

$8 \mathrm{C}$ vs. $8 \mathrm{~B}$ ) or band (Fig. $8 \mathrm{D}$ vs. $8 \mathrm{~B}$ ) in the sinogram indicates that a detector (crystal) element or detector block, respectively, is malfunctioning. That is, it is either not functioning or has a substantially lower sensitivity than the other detectors. In either case, the PMT gain, crystal (detector) map, or photopeak energy window of the affected detector block may need to be adjusted $(11,16)$, the normalization updated, or a faulty hardware component repaired or replaced. As illustrated in Figure 9, sinograms are more visually discriminating than are the reconstructed images for detecting faulty detectors. The integrated software of the PET scanner may also include a routine for quantitative analyses of blank scans and graphical display of the results of these analyses (Fig. 10).

Tomographic uniformity for PET is essentially the same as that for SPECT (Fig. 7C) and may be evaluated with either a ${ }^{18} \mathrm{~F}$-filled cylinder phantom or a ${ }^{68} \mathrm{Ge}$ cylinder source. In practice, the use of a long-lived ${ }^{68} \mathrm{Ge}$ cylinder is preferred because constant refilling of a cylinder with shortlived ${ }^{18} \mathrm{~F}$ is avoided. Tomographic uniformity should be evaluated daily or at least weekly.

In addition to the pronounced differences in sensitivities between direct and cross planes and artifacts between adjoining detector rings (Fig. 11), even optimally perform- ing PET scanners exhibit some further nonuniformity of response. Among the 10,000-20,000 detector elements in a modern ring scanner, slight variations among the detector elements in thickness, light emission properties, electronics performance, and so on result in slightly different line-ofresponse (LOR) counting rates for the same activity. Nonuniform response can be corrected by acquiring data for a uniform flux of annihilation $\gamma$-rays $(11,21)$. Such a scan is known as a normalization scan and the uniformity correction thus derived (analogous to the uniformity correction table of a $\gamma$-camera) is known as a normalization. If $\mathrm{LOR}_{\mathrm{T}}$ is the total number of LORs and a total of $\mathrm{N}_{\mathrm{T}}$ events is acquired in the normalization scan, the average number of counts per LOR, $\overline{\mathrm{N}}_{\mathrm{LOR}}$, is simply:

$$
\overline{\mathrm{N}}_{\mathrm{LOR}}=\frac{\mathrm{N}_{\mathrm{T}}}{\mathrm{LOR}_{\mathrm{T}}}
$$

For the LOR between detectors $\mathrm{i}$ and $\mathrm{j}, \mathrm{LOR}_{\mathrm{ij}}$, with measured number of events $\mathrm{N}_{\mathrm{ij}}$, the normalization factor $\mathrm{NF}_{\mathrm{ij}}$ is:

$$
\mathrm{NF}_{\mathrm{ij}}=\frac{\overline{\mathrm{N}}_{\mathrm{LOR}}}{\mathrm{N}_{\mathrm{ij}}},
$$

and, for the scan of a patient, the normalized, or corrected, number of events, $\mathrm{C}_{\mathrm{ij}}^{\prime}$, in this LOR is:

$$
\mathrm{C}_{\mathrm{ij}}^{\prime},=\mathrm{NF}_{\mathrm{ij}} \mathrm{C}_{\mathrm{ij}} \text {, }
$$

where $\mathrm{C}_{\mathrm{ij}}$ is the raw, or uncorrected, number of events in the LOR between detectors $i$ and $j$.

The normalization scan can be performed using a positronemitting rod source (e.g., ${ }^{68} \mathrm{Ge}$ ) spanning the entire axial FOV and rotating it around the periphery of the FOV, exposing the detector pairs to a uniform photon flux per revolution (as for the daily blank scan). Alternatively, a uniform cylinder of a positron-emitting radionuclide can be scanned and the data thus acquired analytically corrected for attenuation; for a well-defined geometry such as a uniform cylindric source, this correction is straightforward. However, for 3-dimensional PET, the contribution of and correction for scatter with such a large-volume source are nontrivial. In practice, either approach is somewhat problematic because of limited count statistics (and, in the case of 3-dimensional PET, because of scatter). The PET scanner normalization should be created or updated at installation, after major service, whenever deteriorating image quality suggests a new normalization is required, or otherwise at least annually.

Once PET emission data have been corrected for dead time, randoms, system response (by normalization), scatter, and attenuation, the signal per voxel in the reconstructed tomographic images is proportional to the local activity concentration. To make the images quantitative, then, the counting rate per voxel (cps), $\dot{\mathrm{C}}_{\mathrm{ijk}}$, in voxel $\mathrm{ijk}$ should be 

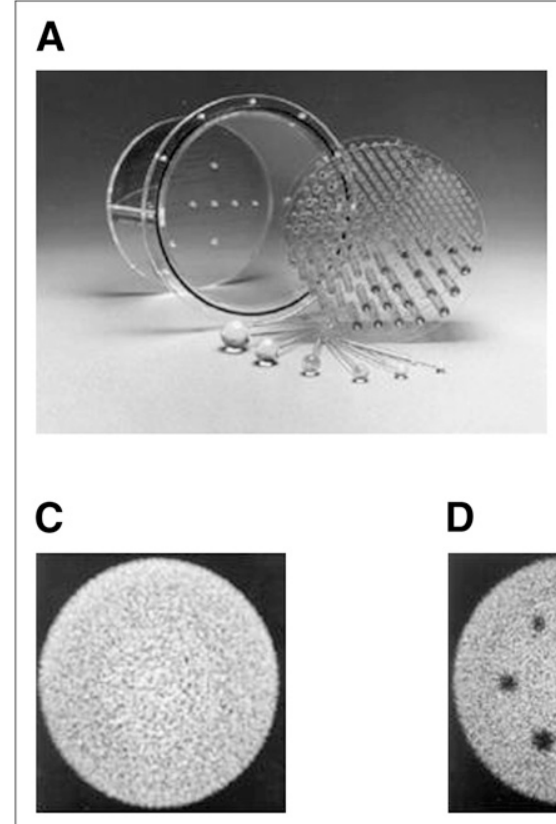

D
B
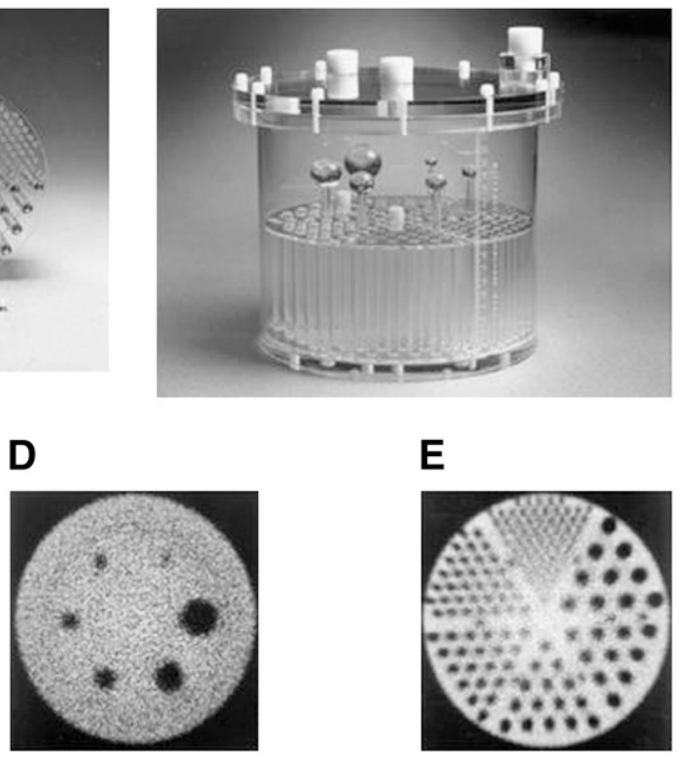

E

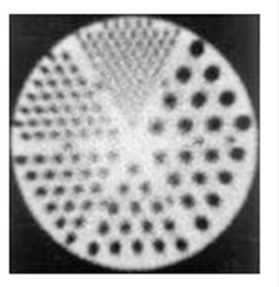

FIGURE 7. Photographs of disassembled (end view) (A) and assembled (side view) (B) phantom (Deluxe Jaszczak Phantom; Data Spectrum Corp.), used for evaluation of overall performance of tomographic imaging systems. This fillable acrylic phantom is $22 \mathrm{~cm}$ in diameter by $19 \mathrm{~cm}$ in length and includes plain section for evaluation of tomographic uniformity $(\mathrm{C})$, section containing empty (cold) spheres ranging from 9.5 to 31.8 $\mathrm{mm}$ in diameter for evaluation of coldsphere contrast (D), and section containing solid (cold) rods ranging from 3.2 to $11.1 \mathrm{~mm}$ in diameter for evaluation of reconstructed spatial resolution $(E)$. Images in C-E have been analytically corrected for attenuation. (Courtesy of Data Spectrum Corp.) divided by the measured system calibration factor ([kBq/ $\mathrm{mL}] /[\mathrm{cps} / \mathrm{voxel}]), \mathrm{CF}$, to yield the activity concentration:

$$
\{\mathrm{A}\}_{\mathrm{ijk}}=\frac{\dot{\mathrm{C}}_{\mathrm{ijk}}}{\mathrm{CF}}
$$

Eq. 10

where $\{\mathrm{A}\}_{\mathrm{ijk}}$ is the activity concentration $(\mathrm{kBq} / \mathrm{mL})$ in voxel ijk. The calibration factor, $\mathrm{CF}$, is derived by the well-counter (or absolute activity) calibration: scanning a calibrated source, that is, a volume source (often a cylinder $\sim 20 \mathrm{~cm}$ in diameter by $\sim 20 \mathrm{~cm}$ in length), with a uniform, welldefined activity concentration at the time of the scan. Implicit in Equation 10 is the assumption that the branching ratios, $\zeta$, of the positron emitter administered to the patient and added to the standard are identical. If not, Equation 10 must be appropriately adjusted:

$$
\{\mathrm{A}\}_{\mathrm{jk}}=\frac{\mathrm{C}_{\mathrm{ijk}}}{\mathrm{CF}} \times \frac{\zeta_{\text {Standard }}}{\zeta_{\text {Patient }}}
$$

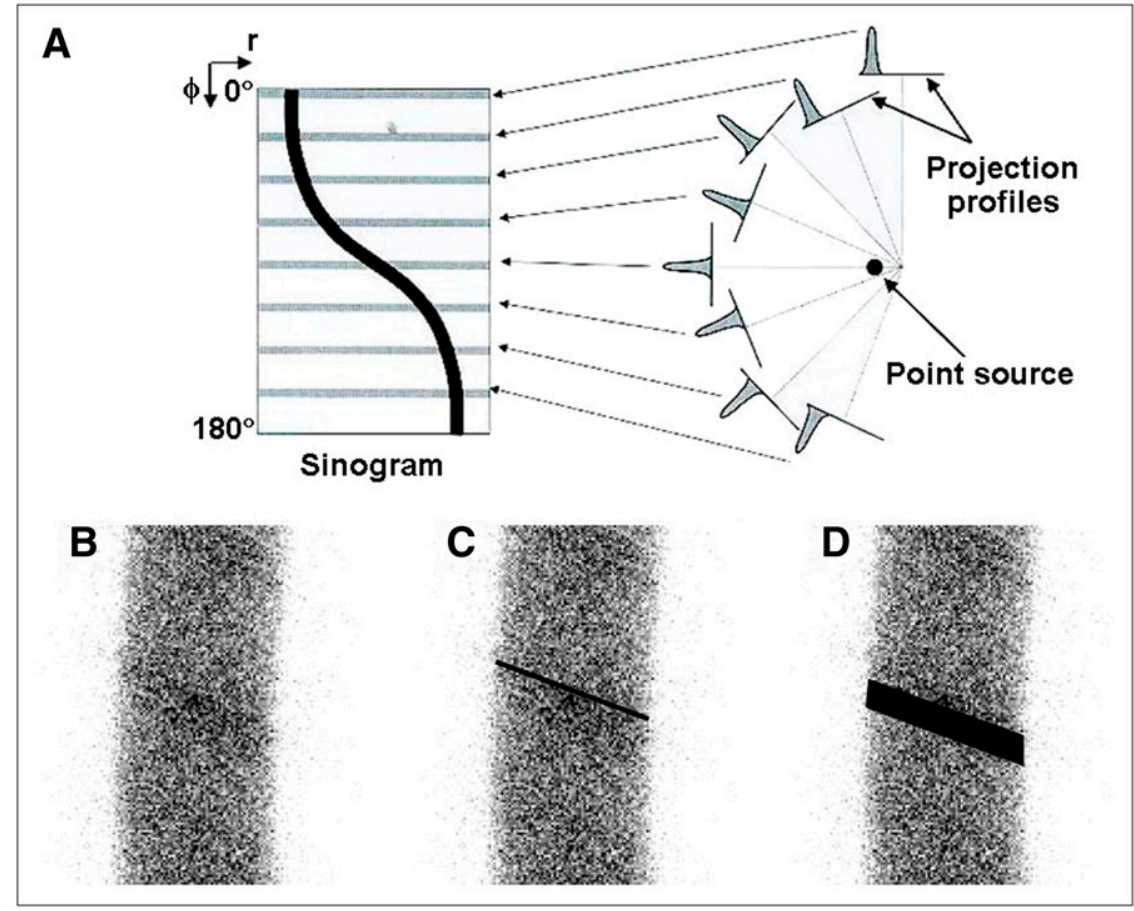

FIGURE 8. (A) Sinogram (i.e., histogram) presentation of emission tomography (i.e., SPECT or PET) data. (B-D) PET sinograms of uniform-cylinder source without any visually perceptible discontinuities or other artifacts (B), with blank diagonal line indicative of faulty detector (crystal) element $(\mathrm{C})$, and blank diagonal band indicative of faulty detector block (D). 
FIGURE 9. Two-dimensional PET sinograms and reconstructed transverse images through hot-sphere ${ }^{18} \mathrm{~F}$-filled phantom: sinogram without any perceptible artifacts (A) and corresponding transverse image (B); sinogram with blank diagonal band, suggestive of faulty detector block (C) and corresponding transverse image (D). Despite obvious defect in sinogram $C$, reconstructed images $B$ and $D$ are virtually indistinguishable. (Courtesy of Dr. Osama Mawlawi, M.D. Anderson Cancer Center, Houston, TX.)
A

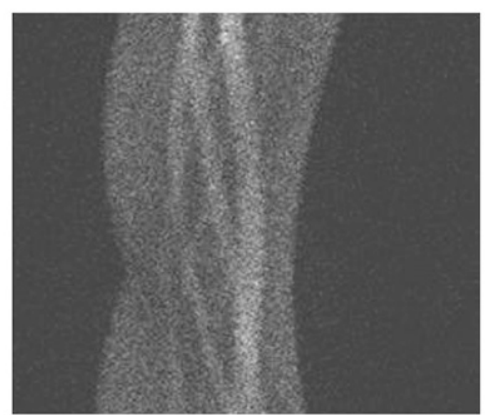

C

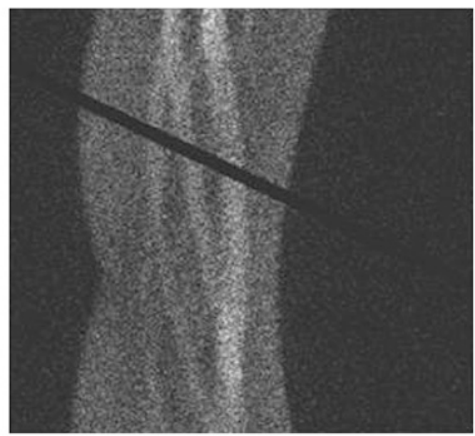

B

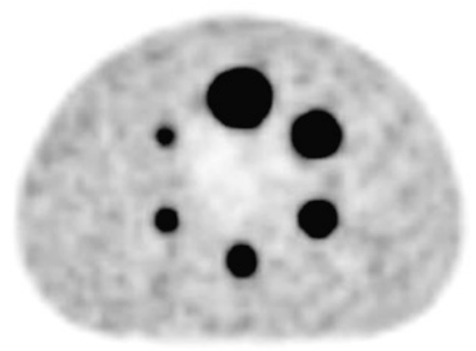

D

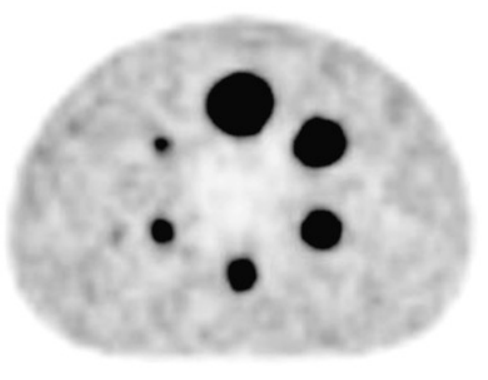

where $\zeta_{\text {Patient }}$ and $\zeta_{\text {standard }}$ are the branching ratios of the positron-emitting isotope administered to a patient and added to the source, respectively. In principle, differences between the geometries of the calibrated source and a patient may introduce some inaccuracy in the determination of activity concentration in situ (Eq. 9 or Eq. 10), but with appropriate attenuation, scatter, and other corrections such inaccuracies should be minimal. The PET scanner wellcounter calibration should be created or updated at installation, after major service, or otherwise at least annually.

\section{CT Scanners}

CT scanners have been in widespread clinical use long before their incorporation into multimodality devices (i.e., SPECT/CT and PET/CT scanners), and detailed protocols for acceptance testing and evaluation of numerous CT performance parameters are well established $(1,2,22)$. As with SPECT and PET scanners, however, less rigorous and less extensive procedures are generally performed for routine CT QC. These QC tests and calibrations apply to the CT subsystems of both SPECT/CT and PET/CT scanners.
FIGURE 10. Proprietary graphical display (GE Healthcare), detector block-bydetector block, of relative values of PET scanner operational parameters derived from blank scan, including coincidence counting rate, singles counting rate, detector dead time, coincidence timing window, and energy setting. Such display allows operator to quickly and easily discern out-of-tolerance results, displayed with grossly different (i.e., lower) intensity than within-tolerance results. (A) Display for acceptable blank scan; that is, blank scan for which all detector parameters are within tolerance. (B) Display for blank scan in which coincidence counting rate, singles counting rate, and dead-time results for 1 block detector are out of tolerance, indicated by black areas (arrows) in respective displays. (Courtesy of Dr. Osama Mawlawi, M.D. Anderson Cancer Center, Houston, TX.)

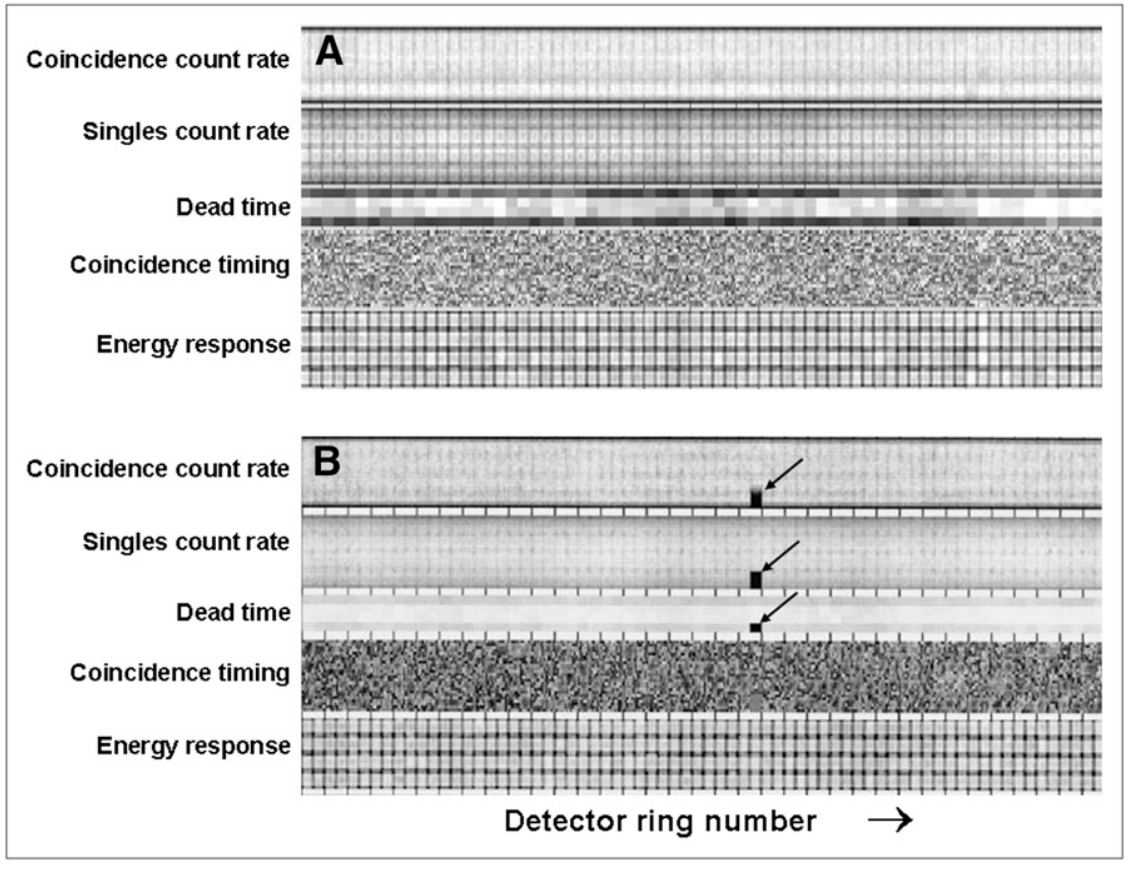




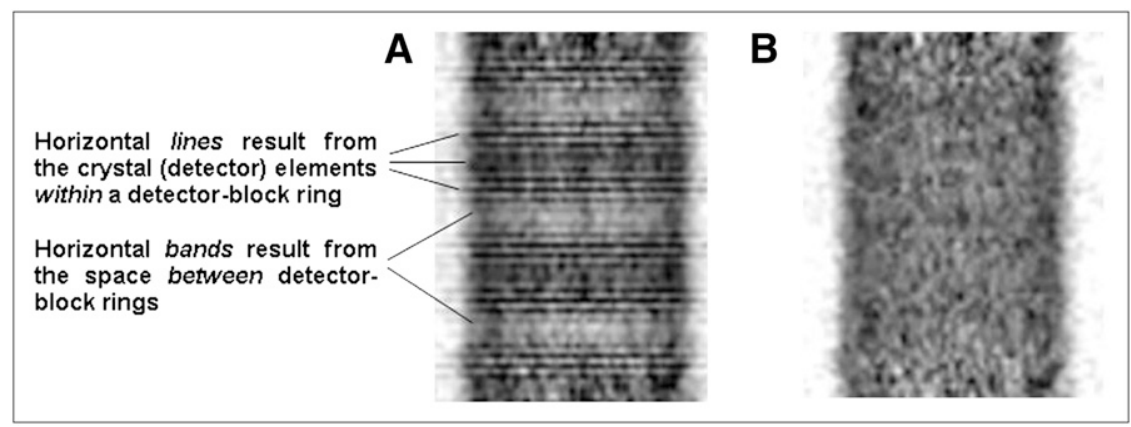

FIGURE 11. Reconstructed coronal images of ${ }^{68} \mathrm{Ge}$ uniform-cylinder phantom without $(A)$ and with $(B)$ normalization applied. (A) Unnormalized (i.e., uncorrected) image has obvious artifacts attributable to differences in sensitivities between direct and cross planes and presence of separate rings of block detectors. (B) Appropriate normalization virtually eliminates these and other artifacts related to nonuniformity of scanner response.

Daily testing of a CT scanner begins with the manufacturer-prescribed x-ray tube warm-up procedure and automatic monitoring, perhaps at various tube voltage $(\mathrm{kVp})$ or current (mA) settings, of the tube output and detector response. Assuming the operator has received the appropriate system-ready message, the daily QC procedures are then performed (12). These include, at a minimum, evaluation of tomographic uniformity, the accuracy of the CT number of water, and image noise (i.e., statistical uncertainty), based on scanning a water-filled cylinder phantom (typically $\sim 20 \mathrm{~cm}$ in diameter by $\sim 20 \mathrm{~cm}$ in length) (Figs. 12A-12D) (10). These parameters should be evaluated using a clinically routine set of scan parameters (i.e., $\mathrm{kVp}, \mathrm{mAs}$, pitch, etc.). Acceptable image uniformity may be confirmed by visual inspection (i.e., by verifying the absence of perceptible ring, streak, or other artifacts) and by quantitative region-of-interest (ROI) analysis of the reconstructed image. In the latter approach (Fig. 12C), $\sim 5$-cm-diameter circular or $\sim 5 \times 5 \mathrm{~cm}$ ROIs $(1$ at the center and 4 at the periphery, at approximately the 12-, 3-, 6-, and 9-o'clock positions) are placed on the image and the mean CT numbers (in Hounsfield units [HU] (23)) compared between the central and each of the peripheral ROIs; the maximum difference should not exceed $5 \mathrm{HU}$. The HU scale is based on a linear transformation of the linear attenuation coefficient as measured by $\mathrm{CT}$, in which the radiodensity of water is assigned a value of $0 \mathrm{HU}$ and that of air at standard temperature and pressure a value of 1,000 HU. For a material $X$ with linear attenuation coefficient $\mu_{X}$, the corresponding $\mathrm{HU}$ value is therefore $\left(\left[\mu_{X}-\mu_{\text {water }}\right] /\right.$

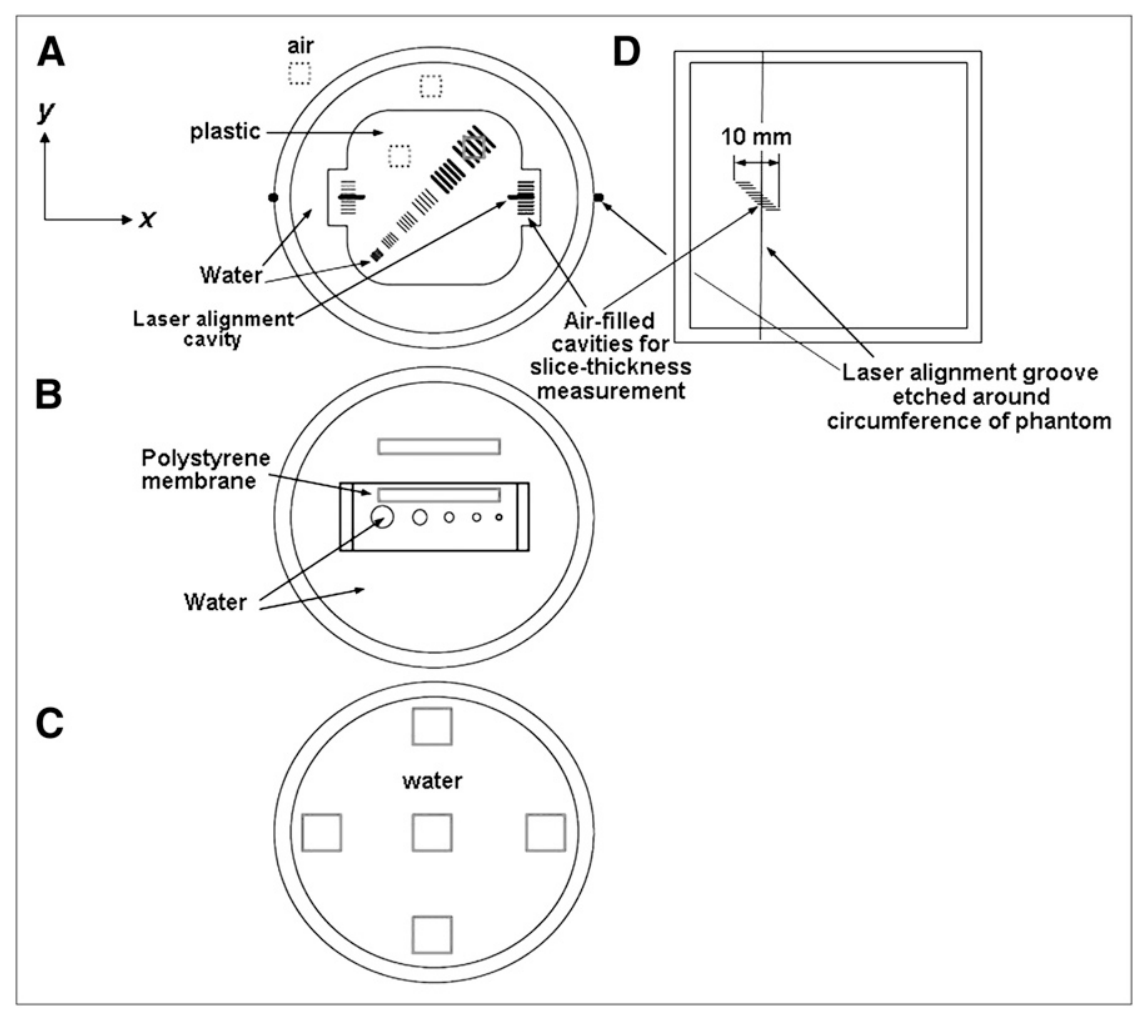

FIGURE 12. Cross-sectional diagrams of GE quality assurance phantom (24) illustrating respective sections (inserts) for evaluation of laser-light alignment, image slice thickness, spatial resolution, linearity, and high-contrast contrast resolution (A); low-contrast uniformity (B); and image uniformity and noise (C). Various short black lines in acrylic insert in section shown in A are cavities that fill with water when phantom is filled, providing high contrast between cavities and acrylic; portion of this section outside this insert, although not shown in black, is also water-filled. Diagonally arranged sets of line cavities ranges are 1.6 (lower left), 1.3, 1.0, 0.8, 0.6, and 0.5 (upper right) $\mathrm{mm}$ in line width and are used to evaluate spatial resolution. Section shown in B includes polystyrene membrane with series of holes $(10,7.5,5,3$, and $1 \mathrm{~mm}$ in diameter) that also fill with water when phantom is filled, providing low contrast between holes and polystyrene. (D) Side-view diagram (not to scale) of section of phantom in A, showing only 1 of slice-thickness measurement components of insert; these line cavities are air-, not water-, filled. They are staggered (offset) $1 \mathrm{~mm}$ apart in longitudinal direction. Also shown in D, as well as in A, is laser-alignment groove around circumference of phantom and 2 corresponding laseralignment cavities. Boxes indicate pertinent ROls for different analyses. (See text for details.) 
$\left.\left[\mu_{\text {water }}-\mu_{\text {air }}\right]\right) \times 1,000$, where $\mu_{\text {water }}$ and $\mu_{\text {air }}$ are the linear attenuation coefficients of water and air, respectively.

The accuracy of the CT number of water and image noise can also be evaluated using the same ROIs. The accuracy of the CT number of water is checked by comparing the mean CT number for each ROI with the CT number, $0 \mathrm{HU}$, expected for water. All of the mean CT numbers thus derived should be within $5 \mathrm{HU}$ of that of water, that is, $0 \pm$ 5 HU. The image noise is evaluated by comparing the SD of the CT number in each of the ROIs with the benchmark, or reference, SD of the scanner, established at the time of its installation: for each ROI, the SD should be no greater than twice the benchmark SD.

In addition to the these daily checks of CT performance, monthly or at least quarterly evaluations of laser alignment, image slice thickness, spatial resolution, linearity (i.e., CT number accuracy), and high- and low-contrast contrast resolution are needed. At the same time, image uniformity and noise, as discussed earlier, should also be evaluated for different tube voltages (i.e., $\mathrm{kVp}$ ) that bracket the range of values used clinically. Evaluation of these parameters requires appropriate phantoms. The Quality Assurance Phantom (GE Healthcare) (24), for example, is a waterfillable polymethylmethacrylate (acrylic) cylinder with multiple sections (i.e., inserts) for measurement of these CT performance parameters (Fig. 12).

These parameters include the following:

- Laser alignment. The laser lights should lie precisely on the circumferential surface groove of the phantom. If the lasers are properly aligned, the 2 laser alignment cavities in the reconstructed transverse image will be horizontal and at precisely the same $y$-position coordinate (Figs. 12A and 12D).

- Slice thickness. Because the line cavities in each of the 2 slice-thickness measurement components of the insert are staggered (offset) $1 \mathrm{~mm}$ apart in the longitudinal direction (Figs. 12A and 12D), the number of black lines (using a high-contrast display) appearing on a transverse reconstructed image corresponds to the slice thickness in millimeters. However, if 1 of the lines appears as gray rather than black, the slice thickness in millimeters equals the number of black lines plus one half, with $0.5 \mathrm{~mm}$ of slice thickness contributed by the cavity appearing as the gray line.

- Spatial resolution. Analogously to assessment of $\gamma$-camera resolution using a 4-quadrant bar phantom (Fig. 4), spatial resolution is assessed by identifying the narrowest set of line cavities in which the separate cavities can be visualized (Fig. 12A).

- Linearity. Linearity (i.e., CT number accuracy) is checked by verifying that the mean CT number (in $\mathrm{HU})$ in a $10 \times 10 \mathrm{~mm}$ ROI in air, water, and acrylic (the dotted squares in Fig. 12A) matches the expected CT number, -1,000, 0, and $100 \mathrm{HU}$, respectively.
- High-contrast contrast resolution. High-contrast contrast resolution is expressed as the SD of the CT number (in $\mathrm{HU})$ in a $10 \times 10 \mathrm{~mm}$ ROI centered over the coarsest set of line cavities (i.e., the set in which the lines are $1.6 \mathrm{~mm}$ in width) (Fig. 12A). The higher the image contrast, the larger the difference between the water and the acrylic CT numbers within this ROI and the higher the SD of the CT number. According to information published by GE Healthcare (24), the acceptable value of the SD of the CT number in this ROI is $37 \pm 4 \mathrm{HU}$ (i.e., 33-41 HU).

- Low-contrast contrast resolution. Low-contrast contrast resolution is evaluated using the insert and the ROIs (boxes, Fig. 12B), $\sim 400 \times 400 \mathrm{~mm}$ in area (Fig. $12 \mathrm{~B})$, and is expressed as the percentage difference between the CT numbers (in HU) in the water and the polystyrene membrane ROIs. According to information published by GE Healthcare (24), this difference should agree within $0.1 \%$ ( $1 \mathrm{HU}$ ) with the benchmark, or reference, low-contrast contrast resolution of the scanner, established at the time of its installation. Optionally, low-contrast resolution can also be evaluated as a function of object size by calculating the foregoing parameter for each of the circular holes in the polystyrene membrane (Fig. 12B).

Daily and monthly (or quarterly) image uniformity and noise can be evaluated using the uniform (plain) section of the GE quality assurance phantom. The red boxes in Figure $12 \mathrm{C}$ illustrate the $5 \times 5 \mathrm{~cm}$ ROIs used for these analyses, as discussed earlier.

Finally, on at least an annual basis, radiation dose should be evaluated by a health physicist or a medical physicist by measuring the CT dose index (CTDI) for various scan parameters (i.e., $\mathrm{kVp}$ and $\mathrm{mA}$ ) which bracket the range of values used clinically. The CTDI is the basic radiation dose parameter in CT and is defined as the integral under the exposure or absorbed dose profile along the patient's longitudinal axis for a single tomographic image (25-27). The volume CTDI (CTDI $\left.{ }_{\mathrm{vol}}\right)$ is derived from the CTDI and is the average dose delivered to a scan volume (vol) for a specific examination. The $100-\mathrm{mm}$ CTDI $\left(\mathrm{CTDI}_{100}\right)$ is the integral under the exposure or absorbed dose profile along a $100-\mathrm{mm}$ length of the patient's longitudinal axis. The weighted $100-\mathrm{mm}$ CTDI $\left(\mathrm{CTDI}_{\mathrm{w}}\right)$ is the weighted average of the $\mathrm{CTDI}_{100}$ measurements at the center and periphery of a dose-measurement phantom:

$$
\mathrm{CTDI}_{\mathrm{w}} \equiv\left[2 / 3 \mathrm{CTDI}_{100}(\mathrm{p})+1 / 3 \mathrm{CTDI}_{100}(\mathrm{c})\right] \cdot \mathrm{f},
$$

Eq. 12

where $\mathrm{CTDI}_{100}(\mathrm{p})$ is the $\mathrm{CTDI}_{100}$ at the periphery (p) of a cylindric phantom, $\mathrm{CTDI}_{100}$ (c) is the $\mathrm{CTDI}_{100}$ at the center (c) of a cylindric phantom and $\mathrm{f}$ is the exposure-to-absorbed dose conversion factor $(33.7 \mathrm{~Gy} / \mathrm{C} / \mathrm{kg}$, or $0.87 \mathrm{rad} / \mathrm{R}$ ) for water or soft tissue. 
Ionization chamber in a peripheral measurement position

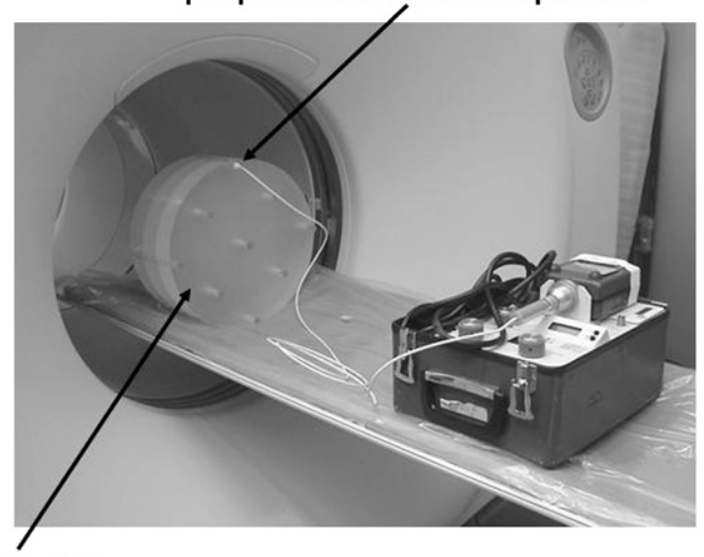

A 32-cm "body"

acrylic phantom

FIGURE 13. Setup for measurement of CT radiation exposures using ionization chamber and $32-\mathrm{cm}$-diameter acrylic torso (body) phantom. (Courtesy of Dr. Sadek Nehmeh, Memorial Sloan-Kettering Cancer Center, New York, NY.)

The $\mathrm{CTDI}_{\mathrm{w}}$ thus reflects the mean absorbed dose over the transverse ( $x$ and $y$ ) dimensions of such a phantom and is an approximation of the average radiation dose to the crosssection of a patient. Measurements of the $\mathrm{CTDI}_{100}(\mathrm{p})$ and $\mathrm{CTDI}_{100}$ (c) are typically performed using ionization chambers or thermoluminescent dosimeters positioned in a commercially available soft-tissue-equivalent acrylic phantom, cylindric in shape and either 16 or $32 \mathrm{~cm}$ in diameter, approximating an adult head or torso (body), respectively (Fig. 13). Ionization chambers actually mea- sure exposure, which is then converted to absorbed dose using the $\mathrm{f}$ factor discussed earlier. Thermoluminescent dosimeters, however, yield absorbed dose directly.

\section{SPECT/CT and PET/CT Image Registration}

Multimodality (i.e., SPECT/CT and PET/CT) devices are still fairly new, and so QC procedures for checking the accuracy of image registration and of CT-based attenuation corrections are not yet well established. Image registration may be checked using a phantom with well-delineated point, line, or volume markers that are both SPECT- or PET- and CT-visible (Fig. 14). Each marker, for example, should be fillable with a solution containing both an imageable radionuclide such as ${ }^{99 \mathrm{~m}} \mathrm{Tc}$ (for SPECT) or ${ }^{18} \mathrm{~F}$ (for PET) and a radioopaque contrast agent such as iohexol (Omnipaque; GE Healthcare). Alternatively, sealed reusable markers composed of long-lived ${ }^{57} \mathrm{Co}$ (for SPECT) or ${ }^{68} \mathrm{Ge}$ (for PET) uniformly dispersed in a radioopaque resin or plastic may be used. The advantage of phantoms having point or line markers (Fig. 14A) is that any misregistration (or offset) between registered images with such welldefined foci is readily apparent by visual inspection of their overlay, or fused, display. Although visual assessment may generally suffice, the misregistration may be quantitated as the mean or maximum Euclidean distance (in $\mathrm{mm}$ ), among all the markers, between the positions of each marker in the 2 modalities. Intuitively, the maximum offset distance should be no greater than the FWHM spatial resolution of the higher-resolution modality, which is CT (equivalent to $\sim 1 \mathrm{~mm}$ for a SPECT/CT or PET/CT scanner). However, point and line markers suffer from the disadvantage that their dimensions are typically well below

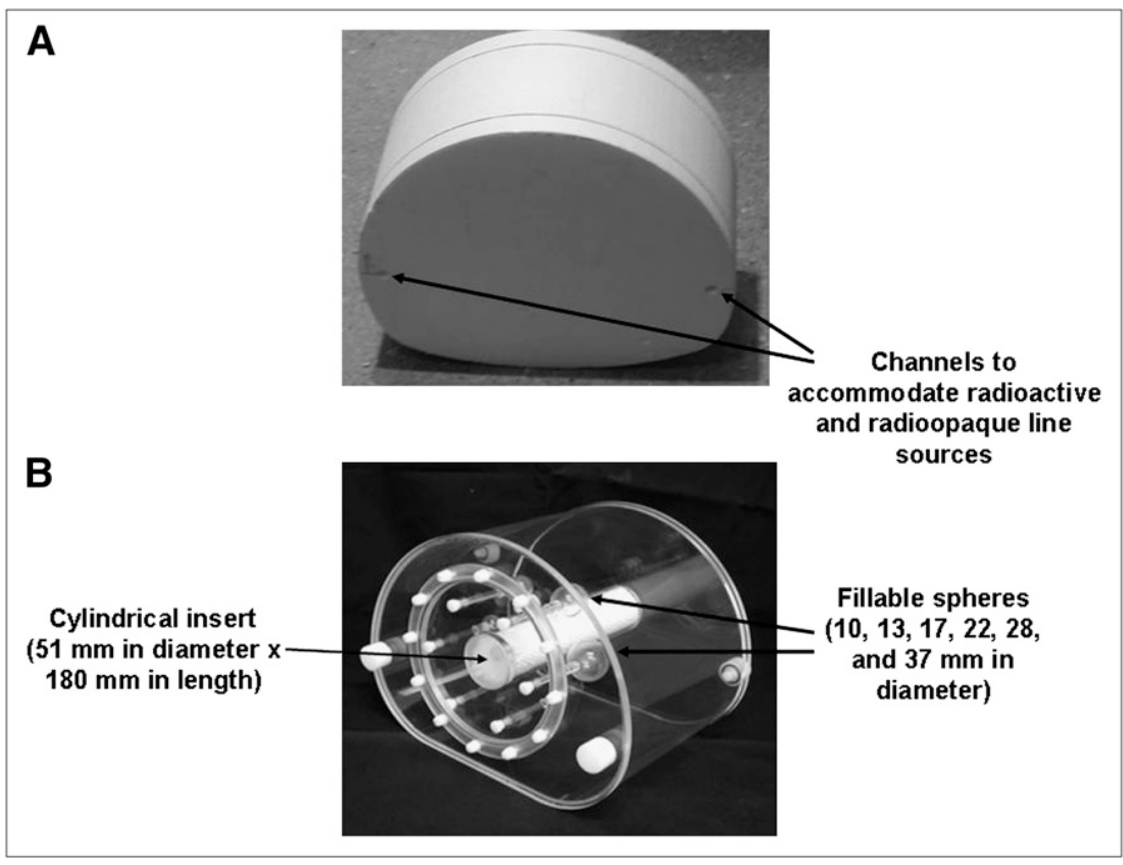

FIGURE 14. Phantoms adaptable to evaluation of accuracy of multimodality image registration. (A) Uniform phantom with 2 channels for line sources. (Courtesy of Dr. Sadek Nehmeh, Memorial Sloan-Kettering Cancer Center, New York, NY.) (B) Phantom (NEMA IEC Body Phantom; Data Spectrum Corp.) with multiple fillable spheres and cylindric insert that can be filled with polystyrene to provide minimally attenuating material, simulating lung in otherwise uniform water-filled volume. 
TABLE 2

Summary of Recommendations for Routine QC of Clinical Nuclear Medicine Instrumentation

\begin{tabular}{|c|c|c|c|c|c|}
\hline Instrument & $\begin{array}{l}\text { At installation or } \\
\text { after repair }\end{array}$ & Annual & Quarterly or monthly & Weekly & Daily \\
\hline Survey meter & Accuracy & Accuracy & $-^{*}$ & $-^{*}$ & $\begin{array}{l}\text { Battery check } \\
\text { Background } \\
\text { Constancy }\end{array}$ \\
\hline Dose calibrator & $\begin{array}{l}\text { Geometry } \\
\text { (volume)- } \\
\text { dependent } \\
\text { response }\end{array}$ & $\mathrm{N} / \mathrm{A}$ & $\begin{array}{l}\text { Linearity } \\
\text { Accuracy }\end{array}$ & $-^{*}$ & Constancy \\
\hline Well counter & $\begin{array}{l}\text { Efficiency } \\
\text { (sensitivity) }\end{array}$ & $\begin{array}{l}\text { Efficiency } \\
\text { (sensitivity) }\end{array}$ & $-^{*}$ & $-^{*}$ & $\begin{array}{l}\text { Energy peaking } \\
\text { Background } \\
\text { Constancy }\end{array}$ \\
\hline Intraoperative probe & $-^{*}$ & $-^{*}$ & $-^{*}$ & $-^{*}$ & $\begin{array}{l}\text { Battery check } \\
\text { Bias check } \\
\text { Energy peaking } \\
\quad \text { (if applicable) } \\
\text { Background } \\
\text { Constancy }\end{array}$ \\
\hline Organ uptake probe & $-^{*}$ & $-^{*}$ & $-^{*}$ & $-^{*}$ & $\begin{array}{l}\text { Energy peaking } \\
\text { Background } \\
\text { Constancy } \\
\text { Efficiency (sensitivity) }\end{array}$ \\
\hline$\gamma$-camera & $\begin{array}{l}\text { References } \\
\text { 3, 5, and } 8\end{array}$ & $-^{*}$ & 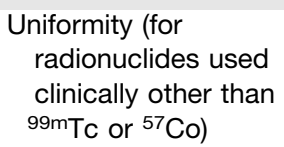 & $\begin{array}{l}\text { Spatial } \\
\text { resolution }\end{array}$ & $\begin{array}{l}\text { Energy peaking } \\
\text { Uniformity } \\
\quad\left(\text { for }{ }^{99 m} \text { Tc or }{ }^{57} \mathrm{Co}\right)\end{array}$ \\
\hline $\begin{array}{l}\text { SPECT scanner or } \\
\text { SPECT subsystem } \\
\text { of SPECT/CT } \\
\text { scanner }\end{array}$ & Reference 6 & $-^{*}$ & $\begin{array}{l}\text { Tomographic } \\
\text { uniformity } \\
\text { Overall system } \\
\text { performance }\end{array}$ & $\begin{array}{l}\text { COR } \\
\text { alignment }\end{array}$ & $\begin{array}{l}\text { Energy peaking } \\
\text { Uniformity } \\
\left.\text { (for }{ }^{99 \mathrm{~m} T c} \text { or }{ }^{57} \mathrm{Co}\right)\end{array}$ \\
\hline $\begin{array}{l}\text { PET scanner or PET } \\
\text { subsystem of } \\
\text { PET/CT scanner }\end{array}$ & $\begin{array}{l}\text { Reference } 4 \\
\text { Normalization } \\
\text { Well-counter } \\
\text { calibration }\end{array}$ & $\begin{array}{l}\text { Normalization } \\
\text { Well-counter } \\
\text { calibration }\end{array}$ & $-^{*}$ & $\begin{array}{c}\text { Tomographic } \\
\text { uniformity }\end{array}$ & Blank scan \\
\hline $\begin{array}{c}\text { CT subsystem of PET/CT } \\
\text { or SPECT/CT scanner }\end{array}$ & $\begin{array}{l}\text { References } \\
1,2 \text {, and } 22\end{array}$ & Dosimetry & $\begin{array}{l}\text { Laser alignment } \\
\text { Image slice } \\
\text { thickness } \\
\text { Spatial resolution } \\
\text { Linearity } \\
\text { High-contrast } \\
\text { resolution } \\
\text { Low-contrast } \\
\text { resolution }\end{array}$ & $-^{*}$ & $\begin{array}{l}\text { Warm-up } \\
\text { Tomographic } \\
\text { uniformity } \\
\text { Accuracy of CT } \\
\text { number of water } \\
\text { Noise }\end{array}$ \\
\hline $\begin{array}{l}\mathrm{PET} / \mathrm{CT} \text { or } \mathrm{SPECT/CT} \\
\text { image registration }\end{array}$ & $-^{*}$ & $-^{*}$ & $\begin{array}{l}\text { Accuracy } \\
\text { CT-based attenuation } \\
\text { corrections }\end{array}$ & $-^{*}$ & $-^{*}$ \\
\hline
\end{tabular}

*Other than QC or performance assessment procedures that may be recommended by manufacturer, no such procedure specifically recommended for this time interval exists.

the spatial resolution of either SPECT or PET scanners and therefore their activities cannot be reliably measured because of partial-volume averaging (28). As a result, the accuracy of the CT-based attenuation corrections cannot be meaningfully assessed. For this purpose, a registration phantom having volume markers with dimensions at least 3 times the SPECT or PET FWHM spatial resolution (to eliminate underestimation of activity due to partial-volume averaging) is preferred (Fig. 14B). For such volume markers, the attenuation-corrected SPECT- or PET-derived activity concentration should be within $10 \%$ of the actual activity concentration in the marker(s) at the time of imaging. Such tests of multimodality image registration should be performed at least monthly.

\section{CONCLUSION}

This article has briefly reviewed, as summarized in Table 2 , routine QC procedures of current nuclear medicine 
instrumentation, including the survey meter, dose calibrator, well counter, intraoperative probe, organ ("thyroid") uptake probe, $\gamma$-camera, SPECT and SPECT/CT scanner, and PET and PET/CT scanner. These procedures and their respective frequencies are presented only as general guidelines. Certainly, there are numerous variations of these procedures that may comprise a sound and compliant QC program.

\section{ACKNOWLEDGMENTS}

We thank Dr. Barbara Binkert of the New York Presbyterian Hospital; Dr. Sadek Nehmeh, Keith Pentlow, Dr. John Humm, and Valerie Longo of Memorial SloanKettering Cancer Center; and Dr. Osama Mawlawi of the M.D. Anderson Cancer Center for their contributions. The author is supported in part by National Institutes of Health (NIH) Small-Animal Imaging Research Program grant R24 CA83084, NIH Center grant P30 CA08748, and NIH grant P01 CA115675-01.

\section{REFERENCES}

1. Lin PJP, Beck TJ, Borras C, et al. Specification and Acceptance Testing of Computed Tomography Scanners. New York, NY: American Association of Physicists in Medicine; 1993.

2. Shepard SJ, Lin PJP, Boone JM, et al. Quality Control in Diagnostic Radiology. College Park, MD: American Association of Physicists in Medicine; 2002.

3. National Electrical Manufacturers Association (NEMA). Performance Measurements of Scintillation Cameras. Rosslyn, VA: NEMA; 2001.

4. National Electrical Manufacturers Association (NEMA). Performance Measurements of Positron Emission Tomographs. Rosslyn, VA: NEMA; 2001.

5. Horton PW, Leach KG, Griffiths JT, et al. The Theory, Specification, and Testing of Anger Type Gamma Cameras. London, U.K.: Hospital Physicists' Association; 1978.

6. Siegel JA, Benedetto AR, Jaszczak RJ, et al. Rotating Scintillation Camera SPECT Acceptance Testing and Quality Control. New York, NY: American Association of Physicists in Medicine; 1987.

7. U.S. Nuclear Regulatory Commission (USNRC). Consolidated Guidance About Materials Licenses: Program-Specific Guidance About Medical Use LicensesFinal Report. NUREG-1556, Vol. 1, Rev. 1. Washington, DC: USNRC; 2005:82.

8. Wegst AV, Ahare A, Correia JA, et al. Scintillation Camera Acceptance Testing and Performance Evaluation. New York, NY: American Association of Physicists in Medicine; 1980.

9. Graham LS, Fahey FH, Madsen MT, van Aswegen A, Yester MV. Quantitation of SPECT performance: report of Task Group 4, Nuclear Medicine Committee. Med Phys. 1995;22:401-409.
10. Nichols KJ, Bacharach SL, Bergmann SR, et al. Instrumentation quality assurance and performance. J Nucl Cardiol. 2006;13:e25-e41.

11. Cherry SR, Sorenson JA, Phelps ME. Physics in Nuclear Medicine. 3rd ed. Philadelphia, PA: Saunders; 2003.

12. Christian P, Waterstram-Rich K, eds. Nuclear Medicine and PET/CT: Technology and Techniques. 6th ed. St Louis, MO: Mosby/Elsevier; 2007.

13. Hasegawa BH, Frenkel A. Physics and history of SPECT/CT. In: Israel O, Goldsmith SJ, eds. Hybrid SPECT/CT: Imaging in Clinical Practice. New York, NY: Taylor \& Francis; 2006:1-24.

14. Townsend DW, Beyer T, Blodgett TM. PET/CT scanners: a hardware approach to image fusion. Semin Nucl Med. 2003;33:193-204.

15. Zanzonico P. Technical requirements for SPECT: instrumentation, data acquisition and processing, and quality control. In: Kramer EL, Sanger JJ, eds. Clinical SPECT Imaging. New York, NY: Raven Press; 1995:7-41.

16. Zanzonico P. Positron emission tomography: a review of basic principles, scanner design and performance, and current systems. Semin Nucl Med. 2004;34: 87-111.

17. Zanzonico P, Heller S. The intraoperative gamma probe: basic principles and choices available. Semin Nucl Med. 2000;30:33-48.

18. Early PJ, Miller WH. Considerations of counting and imaging. In: Early PJ, Sodee DB, eds. Principles and Practice of Nuclear Medicine. 2nd ed. St. Louis, MO: Mosby; 1995:211-214.

19. Saha GB. Fundamentals of Nuclear Pharmacy. New York, NY: Springer-Verlag; 2004.

20. Townsend DW, Bendriem B. Introduction to 3D PET. In: Bendriem B, Townsend DW, eds. The Theory and Practice of $3 D$ PET. Dordrecht, The Netherlands: Kluwer Academic Publishers; 1998:1-10.

21. Bailey D, Gilardi MC, Grootoonk S, et al. Quantitative procedures in 3D PET. In: Bendriem B, Townsend DW, eds. The Theory and Practice of $3 D$ PET. Dordrecht, The Netherlands: Kluwer Academic Publishers; 1998:55-109.

22. American College of Radiology (ACR). ACR technical standard for medical nuclear physics performance monitoring of PET-CT imaging equipment. Available at: http://www.acr.org/SecondaryMainMenuCategories/quality_safety/ guidelines/med_phys/pet_ct_equipment.aspx. Accessed May 20, 2008.

23. Hendee WR. The Physical Principles of Computed Tomography. Boston, MA: Little, Brown and Company; 1983.

24. GE Healthcare. LightSpeed 4.X Lightspeed 16 Technical Reference Manual. CE 0459. Waukesha, WI: General Electric Company; 2005.

25. Gerber TC, Kuzo RS, Morin RL. Techniques and parameters for estimating radiation exposure and dose in cardiac computed tomography. Int J Cardiovasc Imaging. 2005;21:165-176.

26. Morin RL, Gerber TC, McCollough CH. Physics and dosimetry in computed tomography. Cardiol Clin. 2003;21:515-520.

27. Morin RL, Gerber TC, McCollough CH. Radiation dose in computed tomography of the heart. Circulation. 2003;107:917-922.

28. Hoffman EJ, Huang SC, Phelps ME. Quantitation in positron emission computed tomography: 1. Effect of object size. J Comput Assist Tomogr. 1979;3:299-308.

29. Greer K, Jaszczak R, Harris C, Coleman RE. Quality control in SPECT. J Nucl Med Technol. 1985;13:76-85.

30. Johns H, Cunningham J. The Physics of Radiology. Springfield, IL: Charles C. Thomas; 1974. 